\title{
Neurotransmitters Play as a Key Role in Adult Neurogenesis
}

\author{
Vahid Hajali', ${ }^{1,}$, Hamid Reza Moradi ${ }^{3}$, Sajad Sahab Negah ${ }^{2,4 *}$ \\ ${ }^{1}$ Quchan Higher Health Education Center, Mashhad University of Medical Sciences, Mashhad, Iran \\ ${ }^{2}$ Department of Neuroscience, Faculty of Medicine, Mashhad University of Medical Sciences, Mashhad, Iran \\ ${ }^{3}$ Department of Basic Sciences, Faculty of Veterinary, University of Tehran, Tehran, Iran \\ ${ }^{4}$ Shefa Neuroscience Research Center, Khatam Alanbia Hospital, Tehran, Iran
}

Article Info:

Received: 17 Dec 2017

Revised: 11 Apr 2018

Accepted: 26 May 2018

\section{A BSTRACT}

Introduction: New neurons are constantly created in specific areas of the adult brain and functionally integrated into neuronal networks. Neural stem cells exist in specific microenvironments in the brain called the stem cell niches. It has been shown that neurotransmitters provide vital components of the niche signals and modulate several aspects of neurogenesis. It has been demonstrated that changes in neurotransmitter signaling may influence adult neurogenesis. Further works are required to clarify how neurotransmitter signaling pathways control neurogenesis. Conclusion: In this review, we discuss how neurotransmitter signaling regulates the development of new functional neurons. We also review the potential roles of neurotransmitters for cell therapy.

*Corresponding Author: Sajad Sahab Negah

E-mail:sahabnegahs@mums.ac.ir 


\title{
نوروترانسميتر ها نقشى كليدى در نورونزايـى بالغين ايفاء مى كنند
}

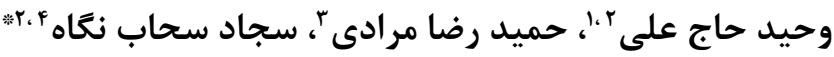 \\ 'مجتمع آموزش عالى سلامت قوجان، دانشكاه علوم بزشكى مشهد، مشهد، ايران

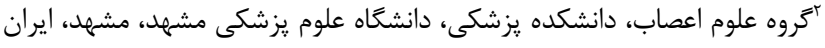

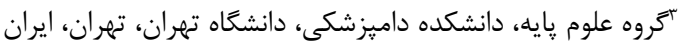

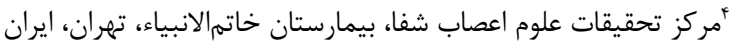

\section{اطلاعات مقاله:}

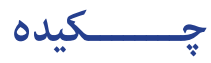

مقدمه: نورونهاى جديد به صورت دائم در مناطق خاصى از مغز بزرگسالان توليد مى شوند و به صورت

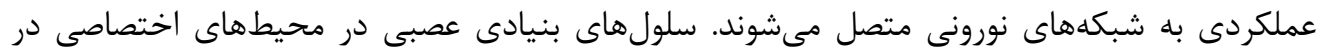

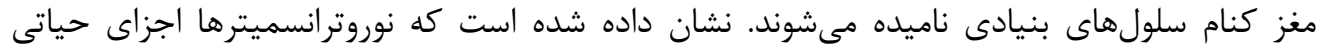

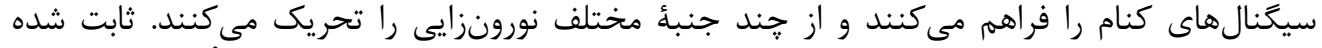

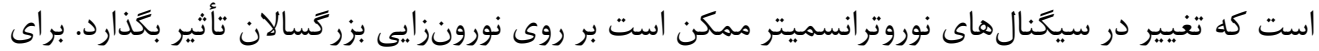

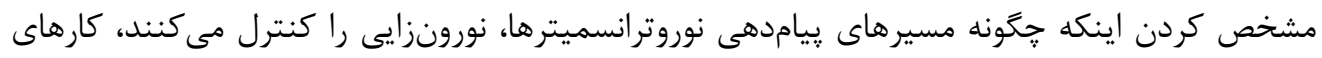

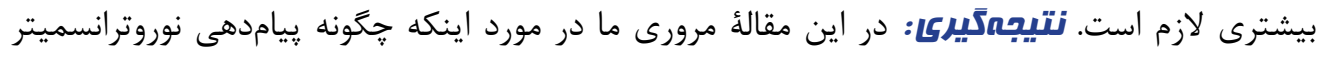

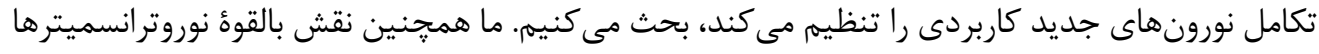
براى سلول درمانى را بررسى جلى مي كنيه.

كليد وازمها:

ا. r

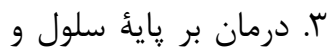




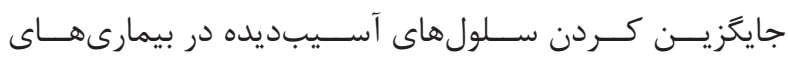

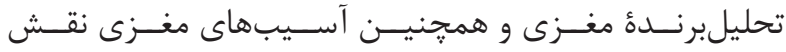

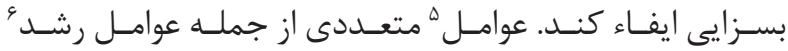

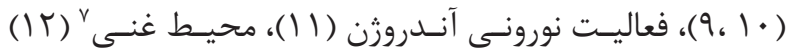

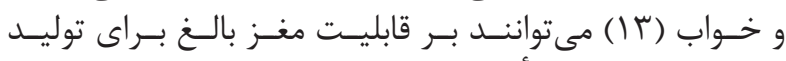

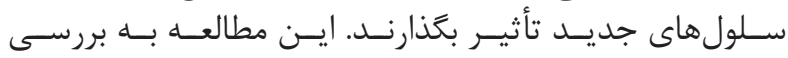

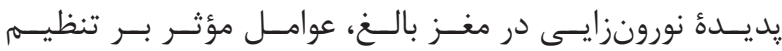

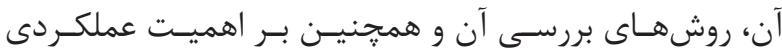

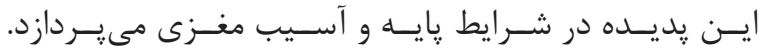

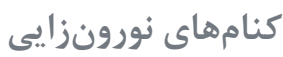

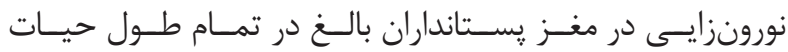

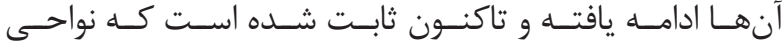

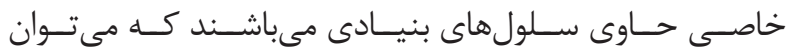

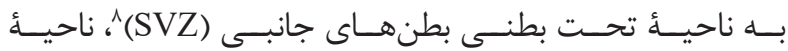

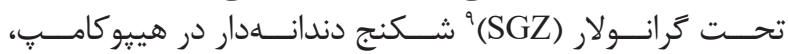

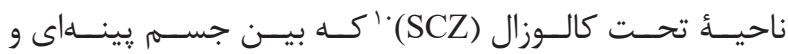

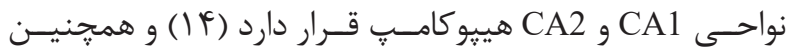

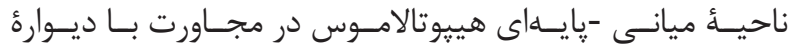

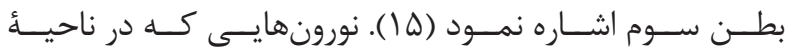

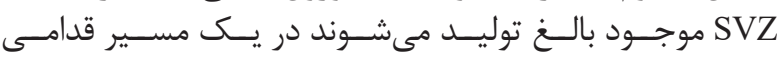

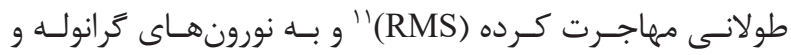

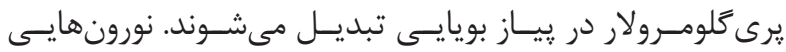

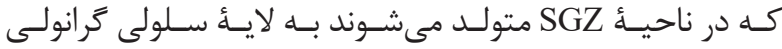

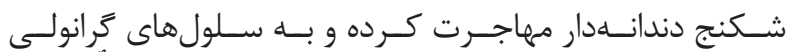

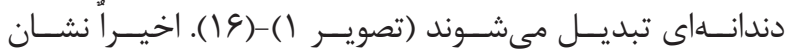

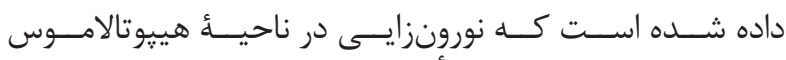

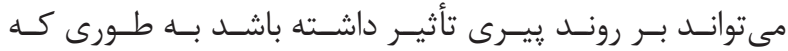

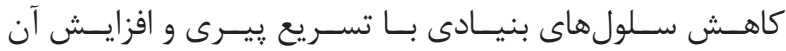

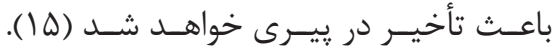

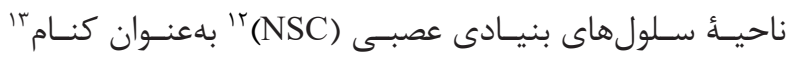

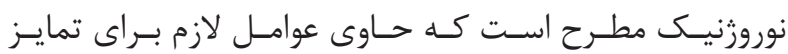

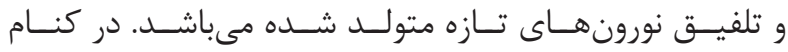

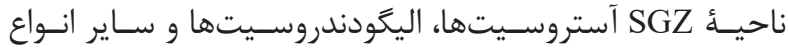

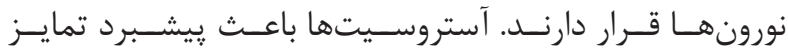

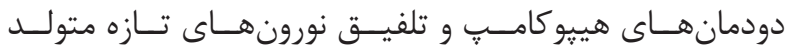

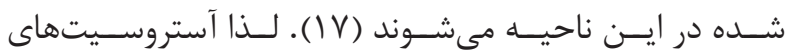

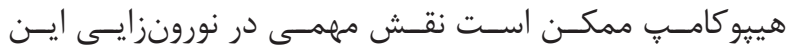

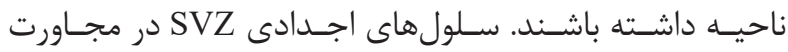

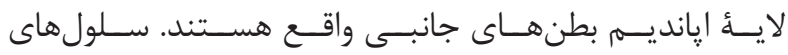

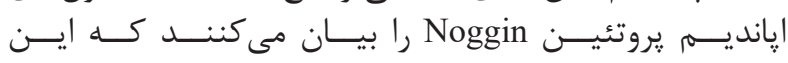

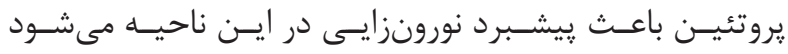

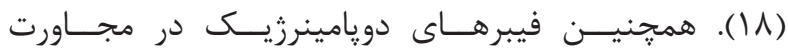

${ }^{1}$ Scientific dogma

${ }^{2}$ Neurogenesis

${ }^{3}$ Central nervous system

${ }^{4}$ Postnatal

${ }^{5}$ Factors

${ }^{6}$ Growth factors

${ }^{7}$ Environmental enrichment

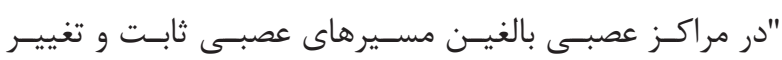

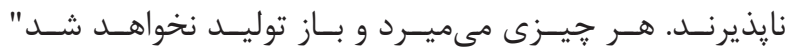

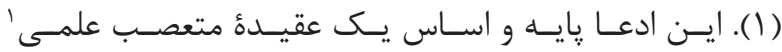

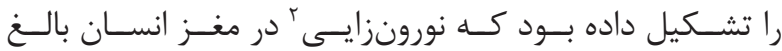

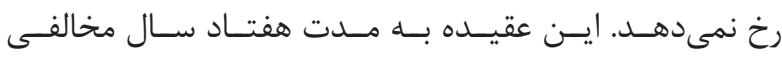

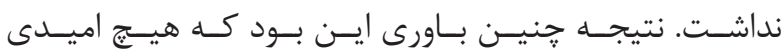

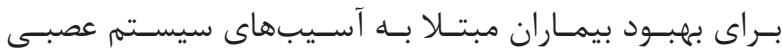

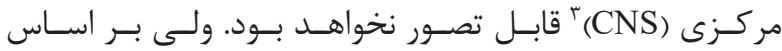

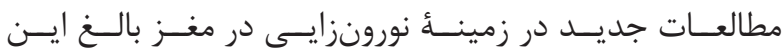

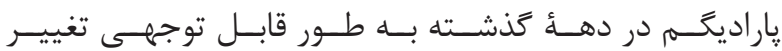

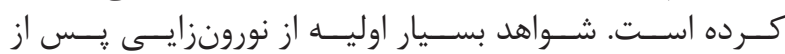

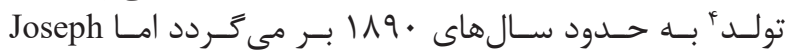

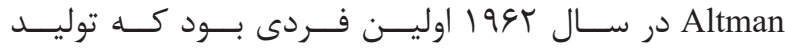

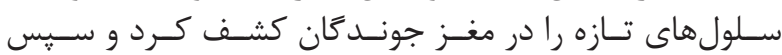

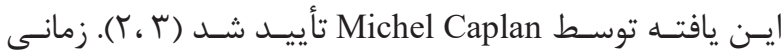

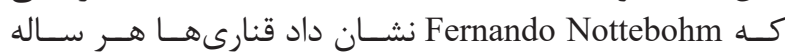

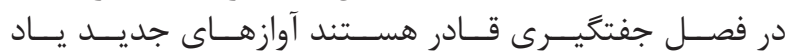

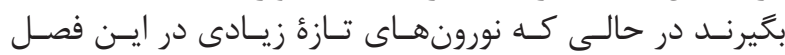

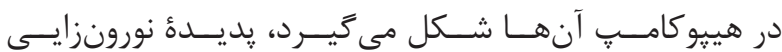

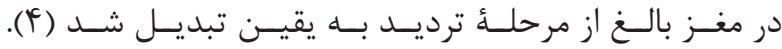

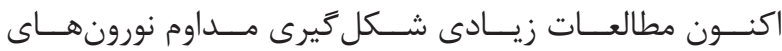

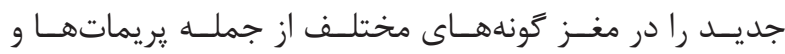

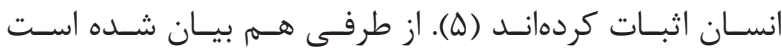

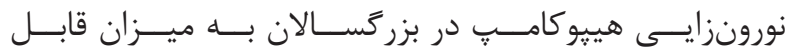

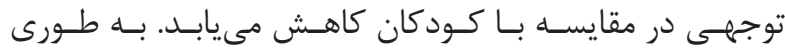

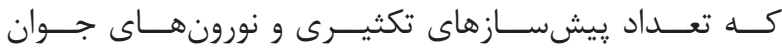

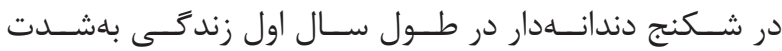

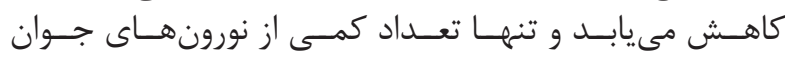

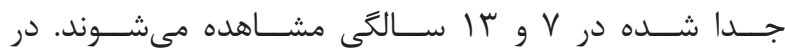

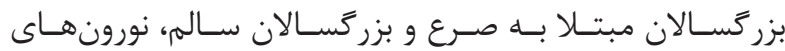

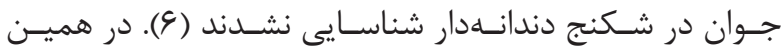

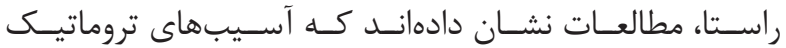

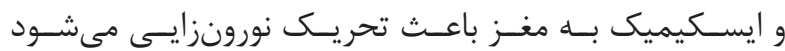

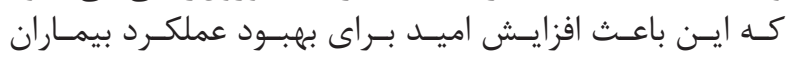

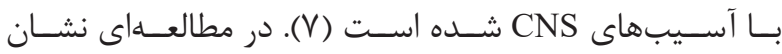

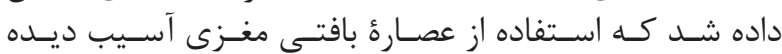

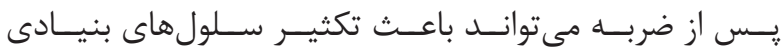

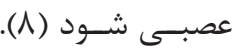

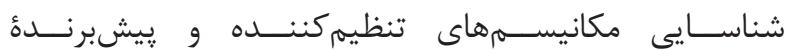

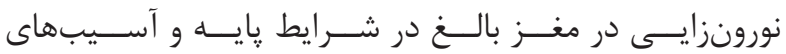

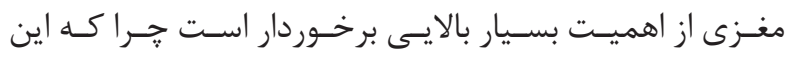

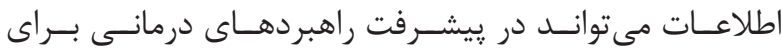

\footnotetext{
${ }^{8}$ Sub ventricular zone

${ }^{9}$ Sub granular zone

${ }^{10}$ Subcallosal zone

${ }^{11}$ Rostral migratory stream

${ }^{12}$ Neural stem cells

${ }^{13}$ Niches
} 


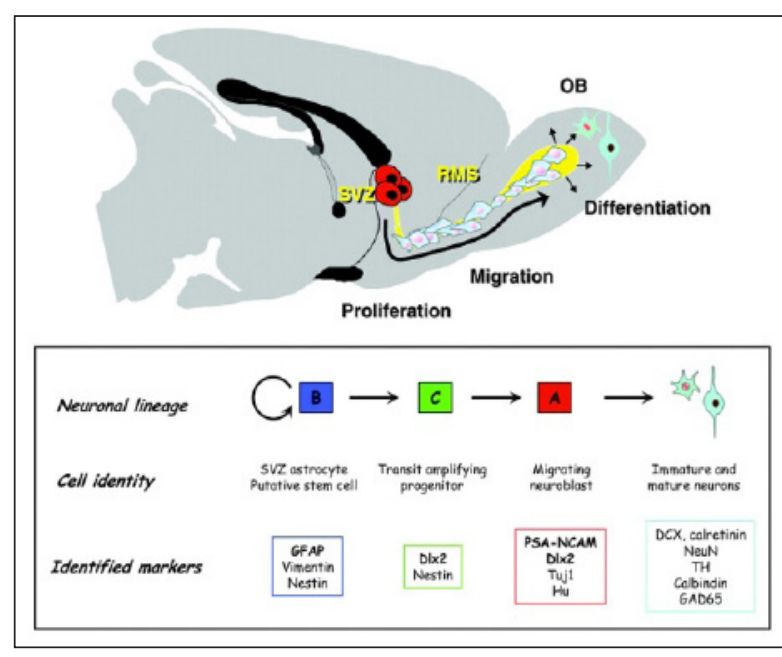

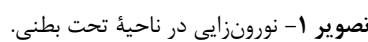

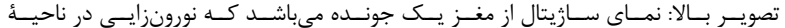

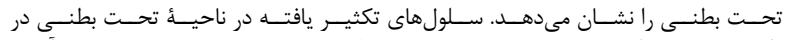

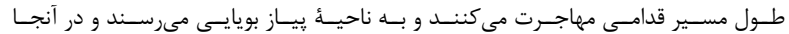

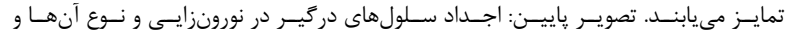

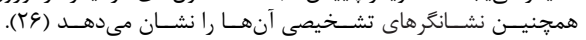

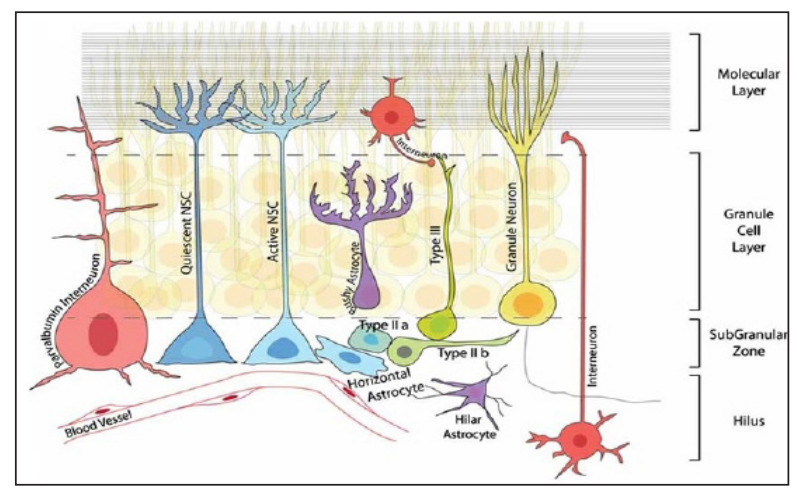

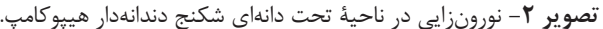

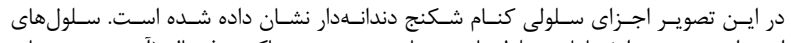

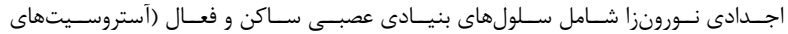

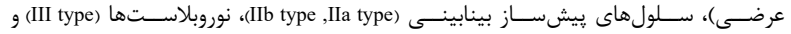

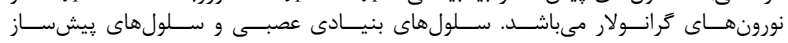

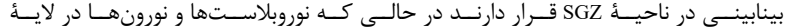

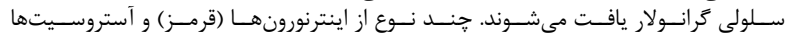

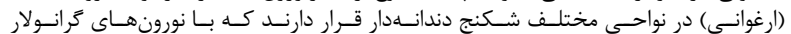

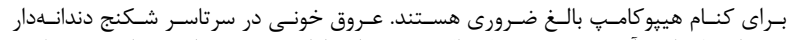

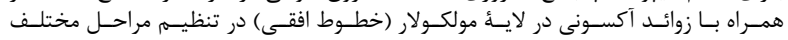

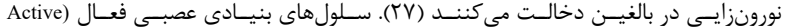

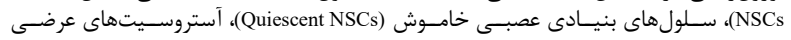
(Horizontal astrocytes)

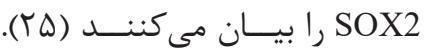
تنظيم نورونزايى در مغز بالغ سالم

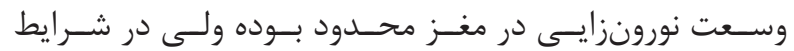

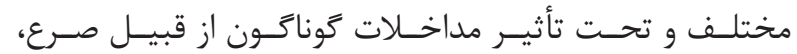

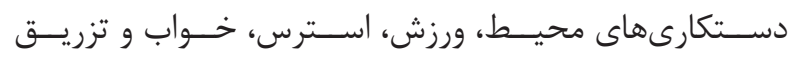

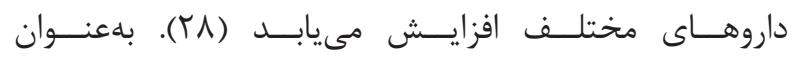

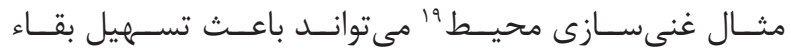

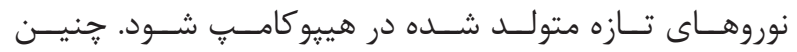

\footnotetext{
${ }^{14}$ Signaling

${ }^{15}$ Vascular endothelial growth factor

${ }^{16}$ Marker
}

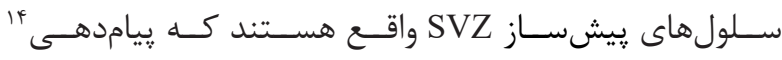

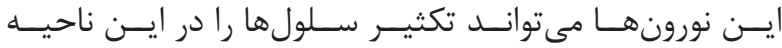

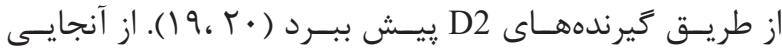

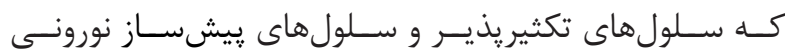

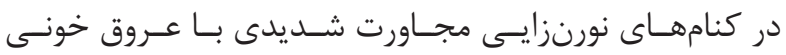

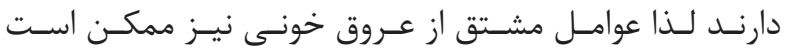

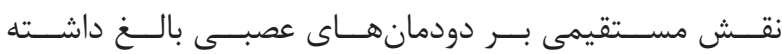

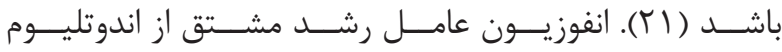

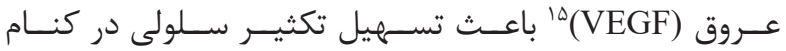

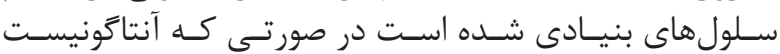

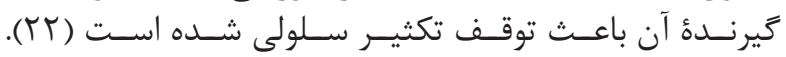

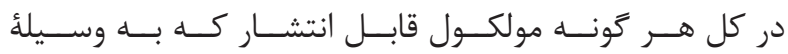

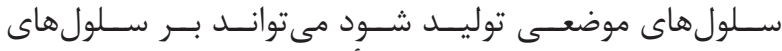

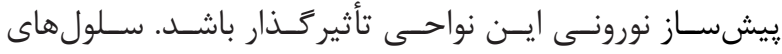

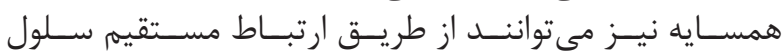

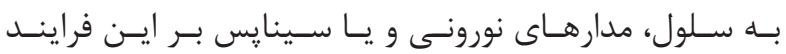

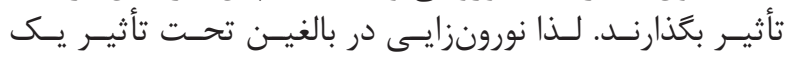

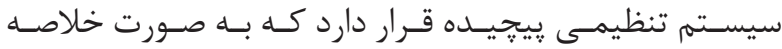

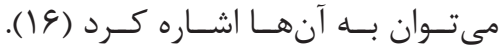

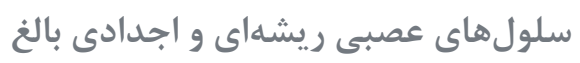

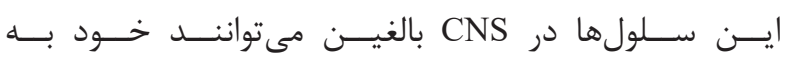

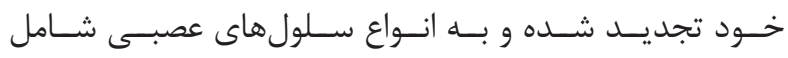

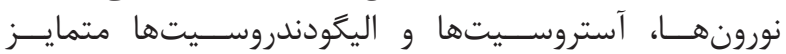

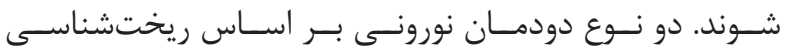

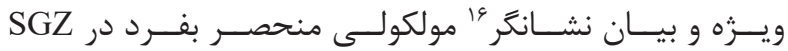

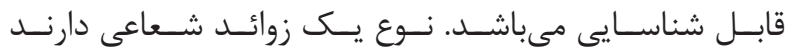

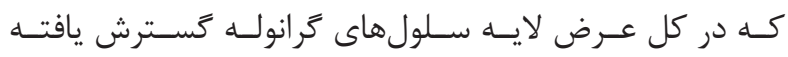

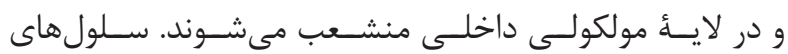

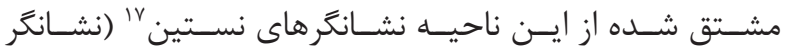

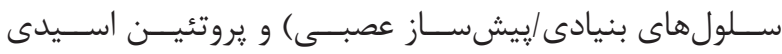

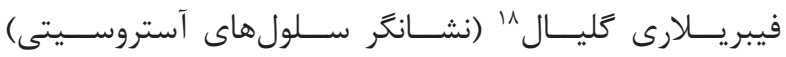

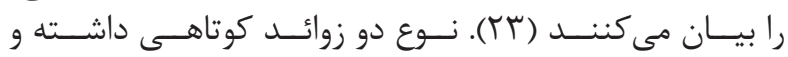

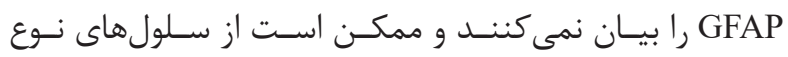

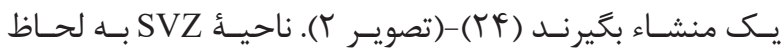

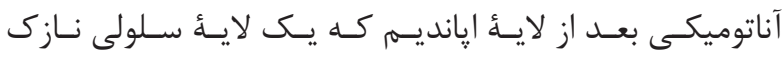

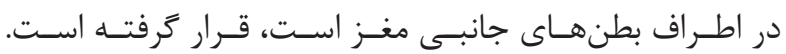

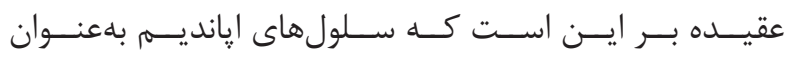

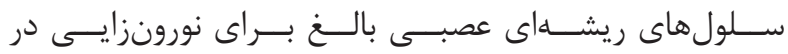

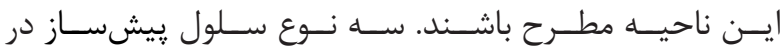

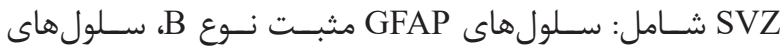

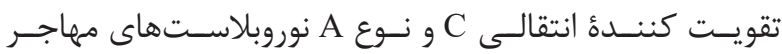

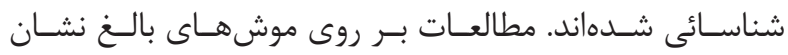

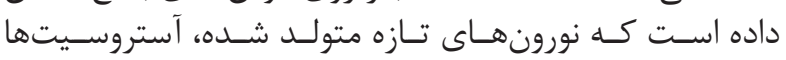

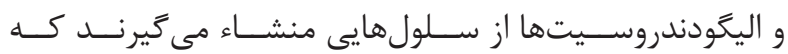

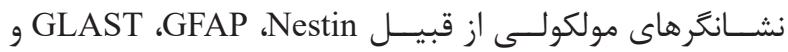

\footnotetext{
${ }^{17}$ Nestin

${ }^{18}$ GFAP (glial fibrillary acidic protein)

${ }^{19}$ Environmental enrichment
} 


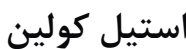

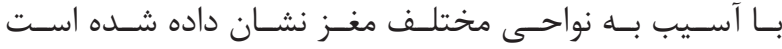

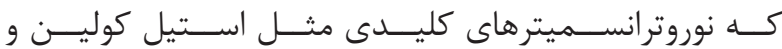

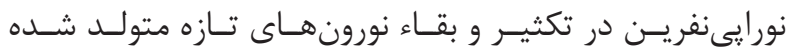

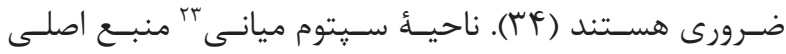

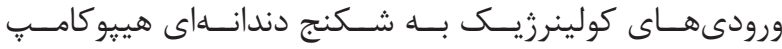

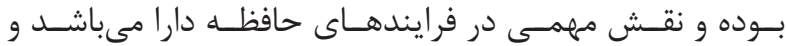

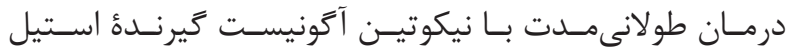

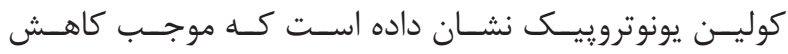

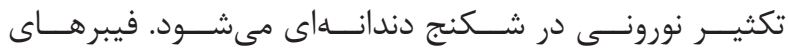

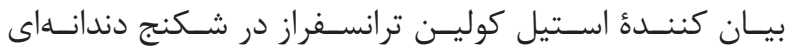

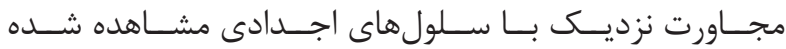

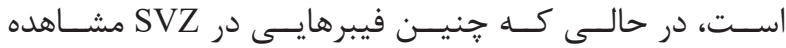

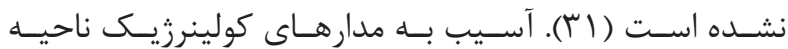

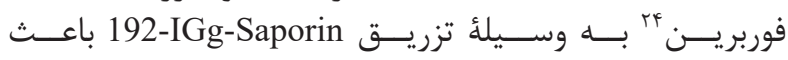

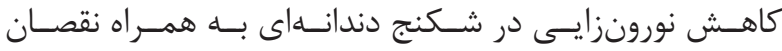

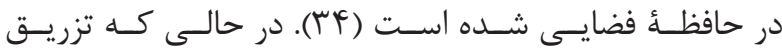

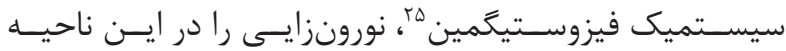

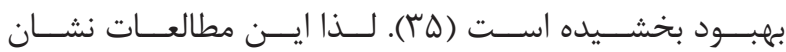

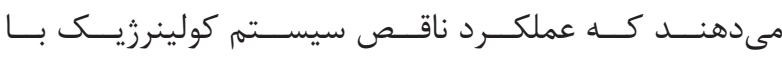

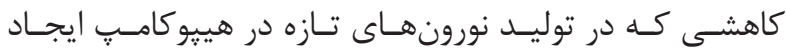

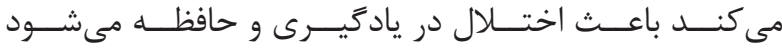

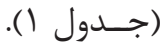
نور آدرنالين

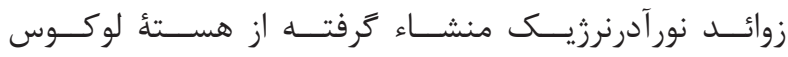

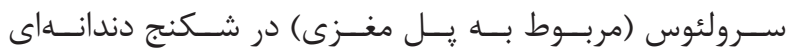

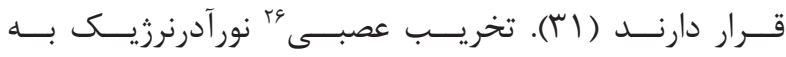

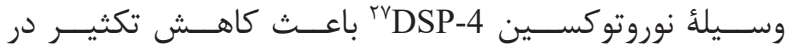

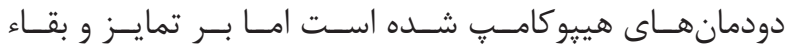

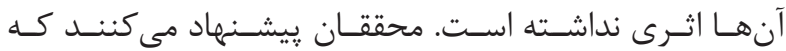

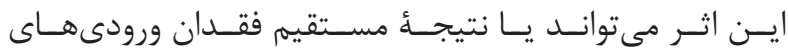

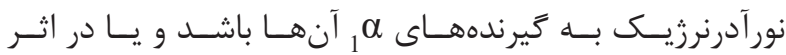

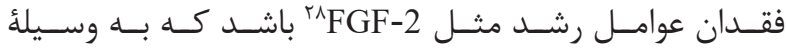

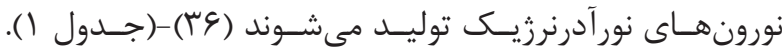

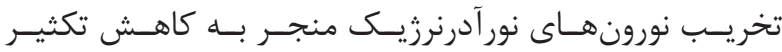

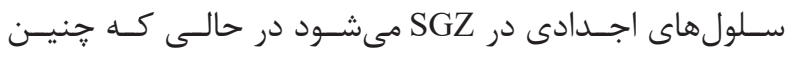

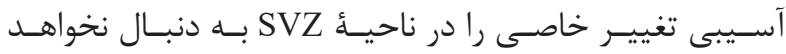

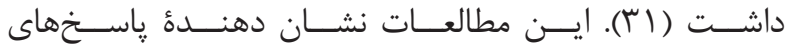

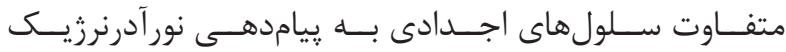

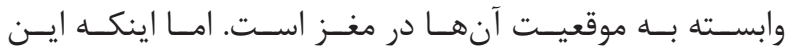

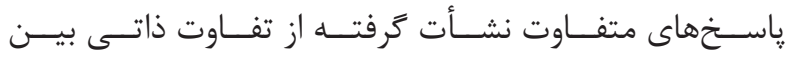

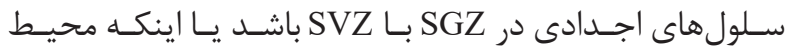

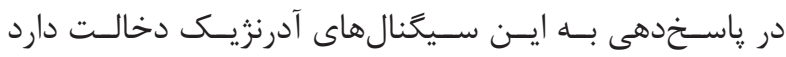

${ }^{20}$ Action potentials

${ }^{21}$ 5-hydroxytryptamine

${ }^{22}$ Selective serotonin reuptake inhibitor

${ }^{23}$ Medial septum

${ }^{24}$ Forebrain

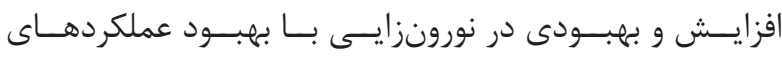

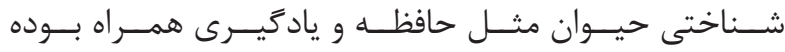

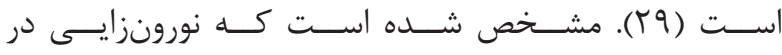

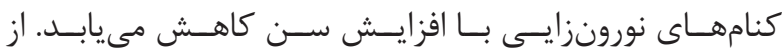

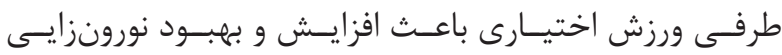

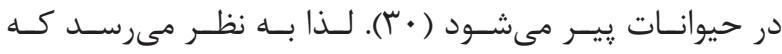

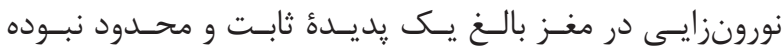

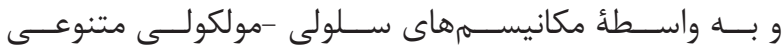

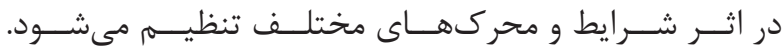

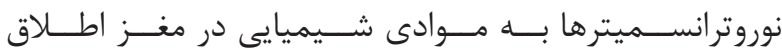

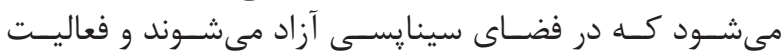

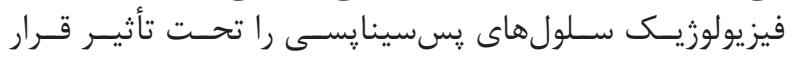

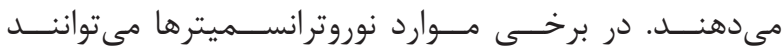

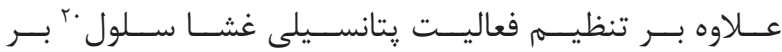

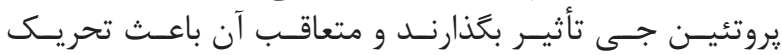

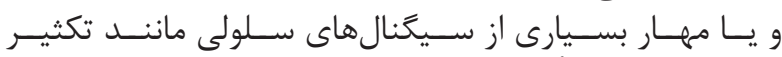

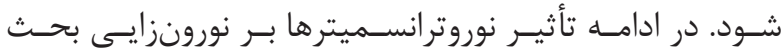

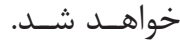
تأثير نوروترانسميترها بر نورونزايى سيى

سروتونين

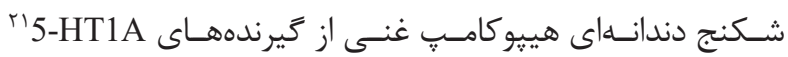

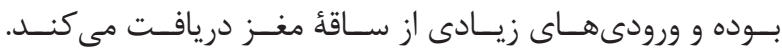

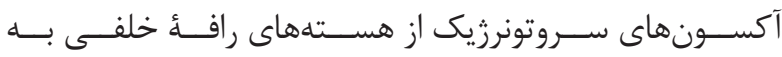

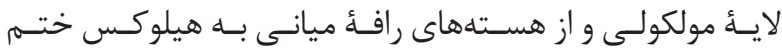

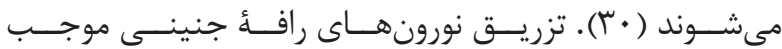

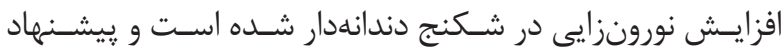

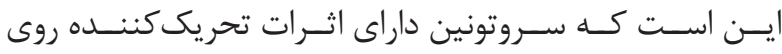

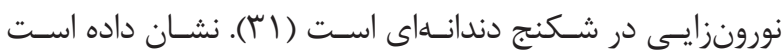

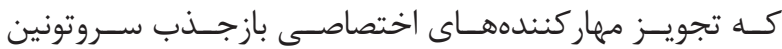
"r (SSRI)

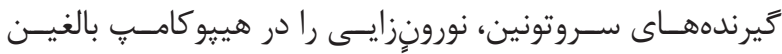

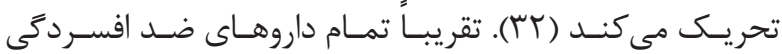

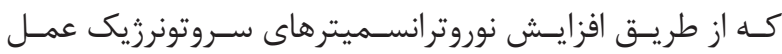

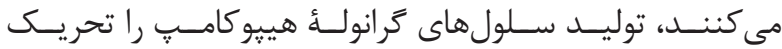

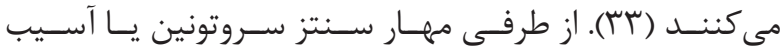

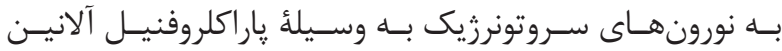

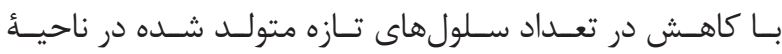

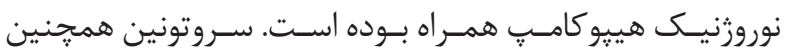

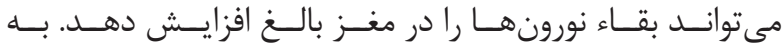

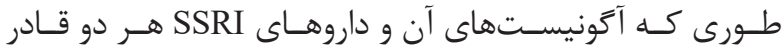

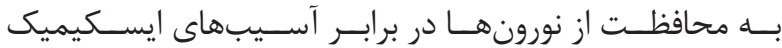

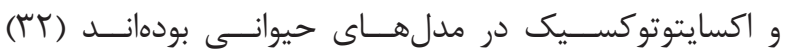

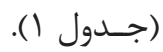

\footnotetext{
${ }^{25}$ Physostigmine

${ }^{26}$ Denervation

${ }^{27}$ Neurotoxin N-(2-chloroethyl)-N-ethyl-2-bromobenzylamine (DSP-4)

${ }^{28}$ Fibroblast growth factor
} 


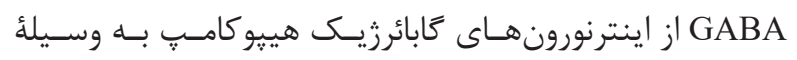

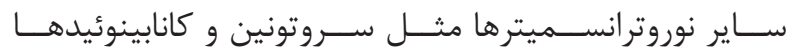

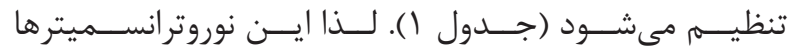

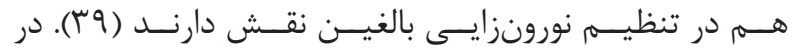

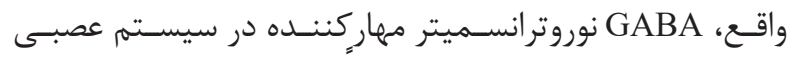

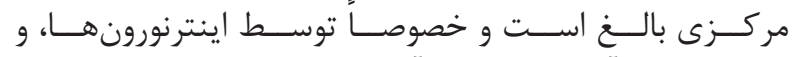

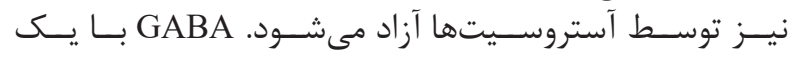

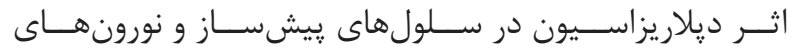

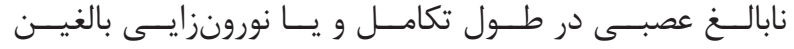

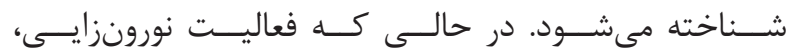

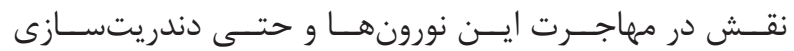

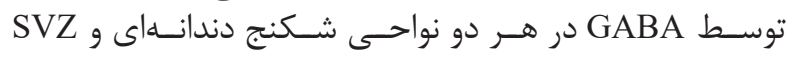

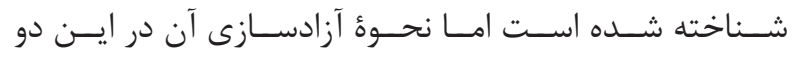

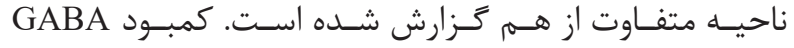

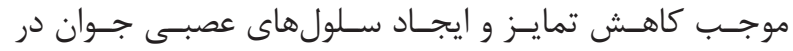

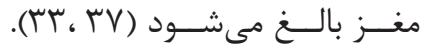

حَلوتامات

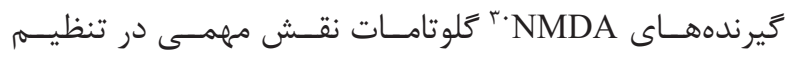

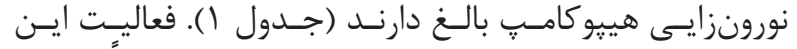

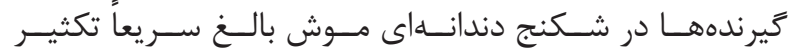

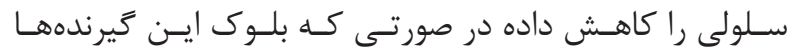

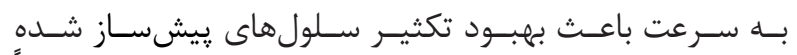

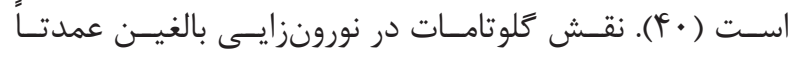

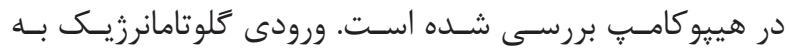

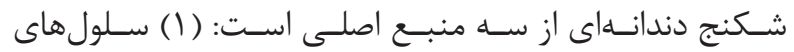

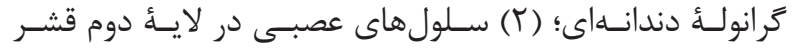

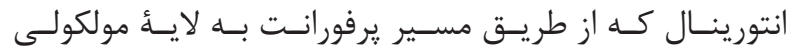

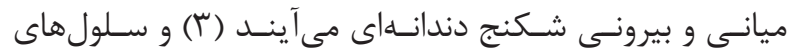

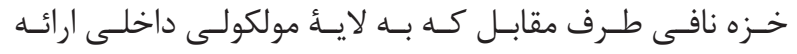

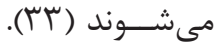

تزريــق حــاد آنتاكونيســت گيرنــده NMDA، MK-801 و و

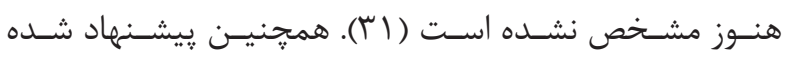

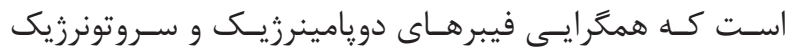

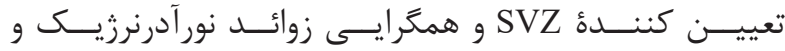

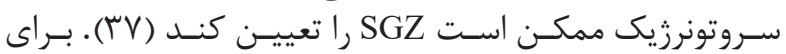

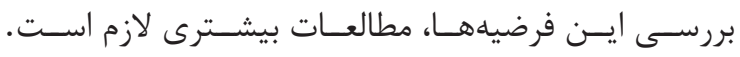
دويامين

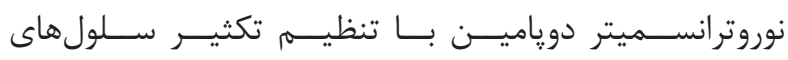

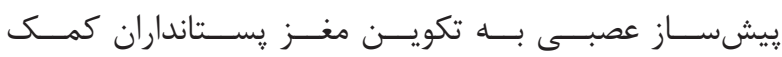

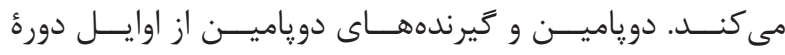

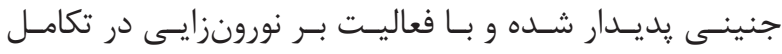

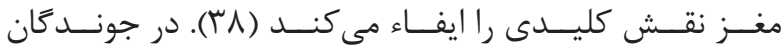

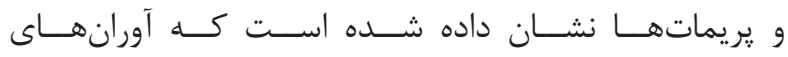

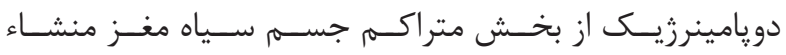

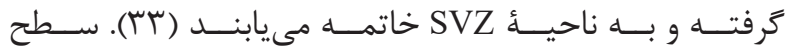

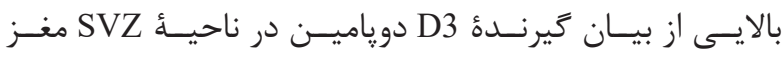

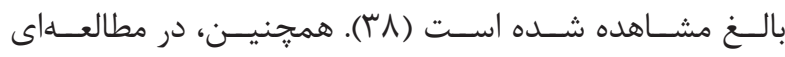

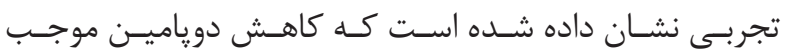

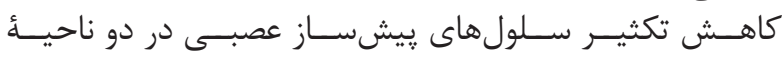

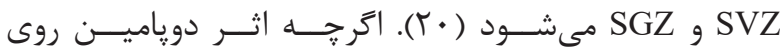

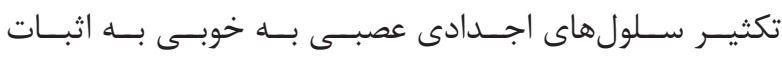

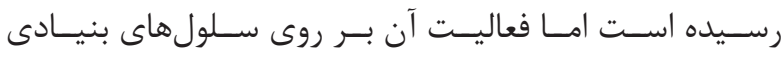

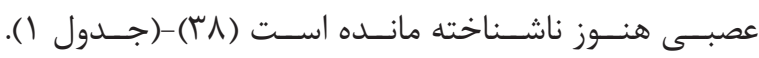

\section{كاما آمينوبوتريك اسيد}

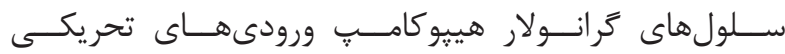

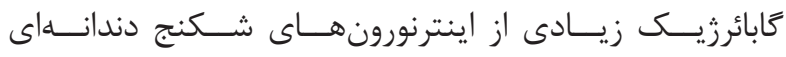

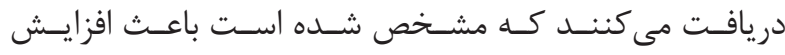

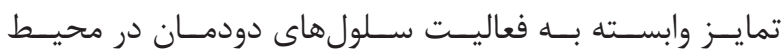

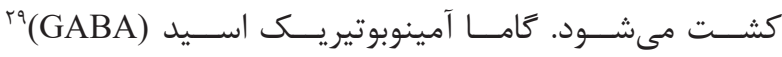

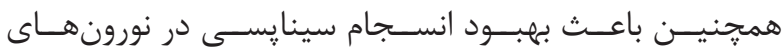

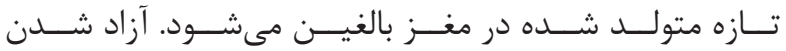

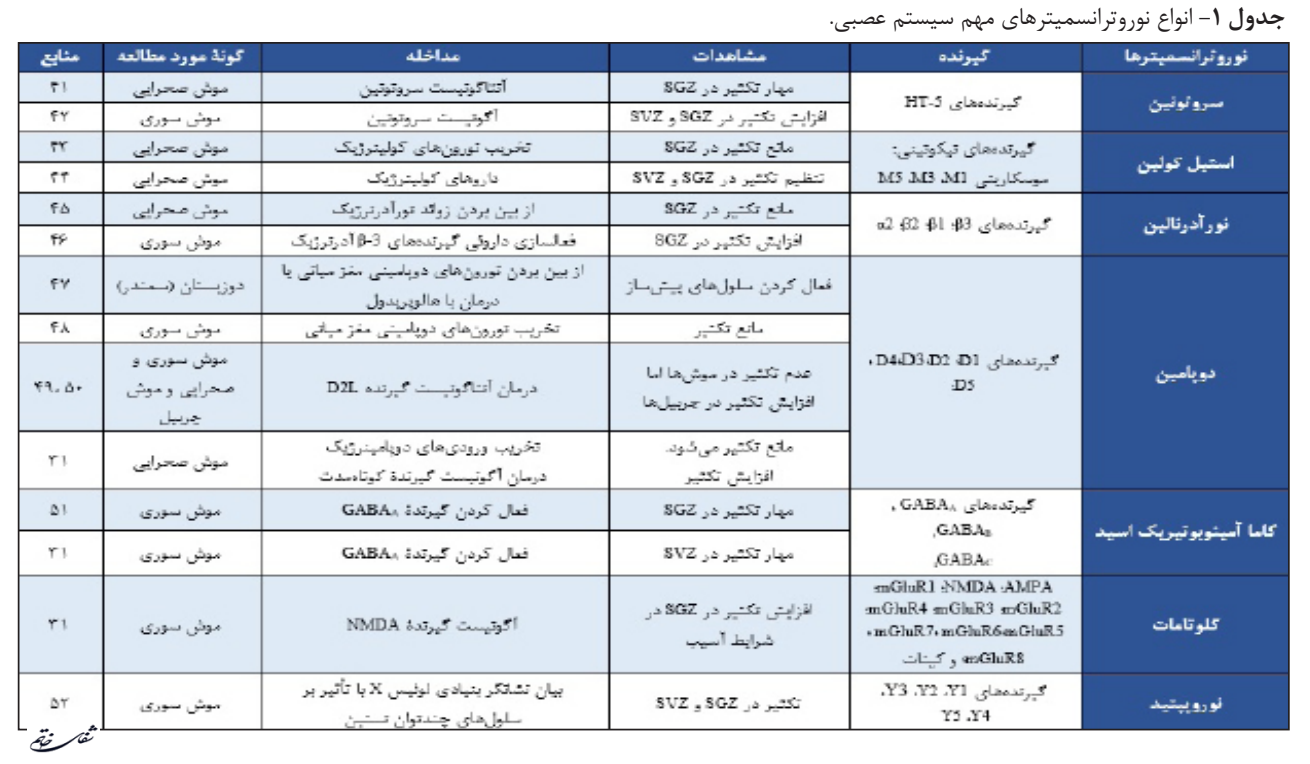




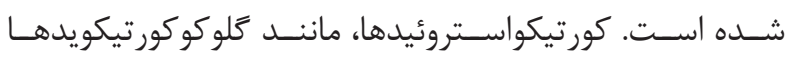

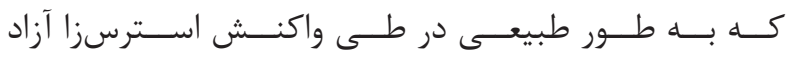

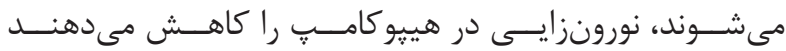

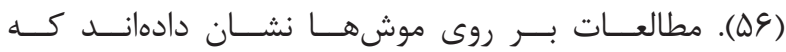

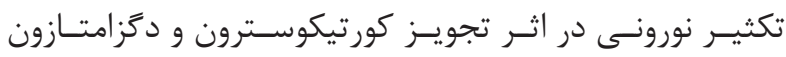

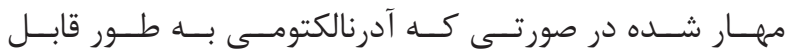

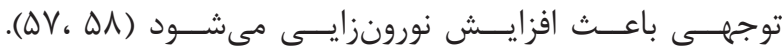

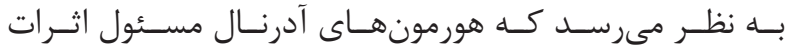

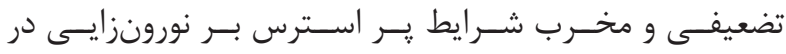

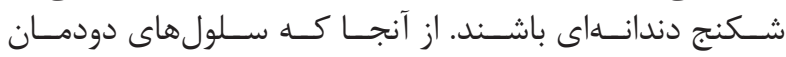

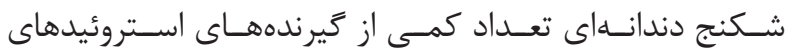

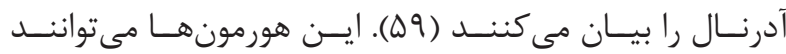

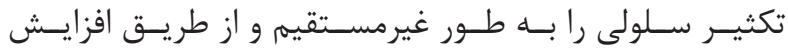

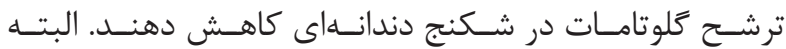

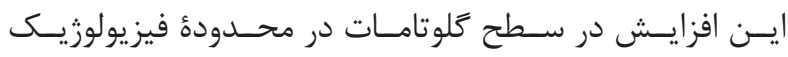

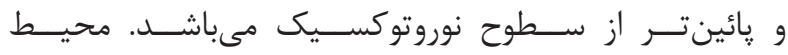

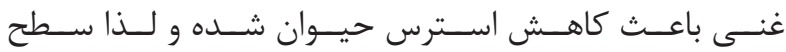

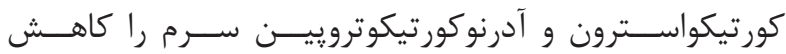

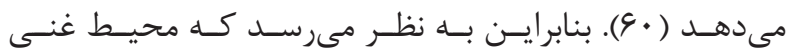

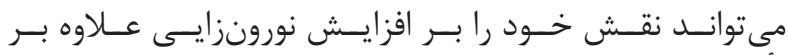

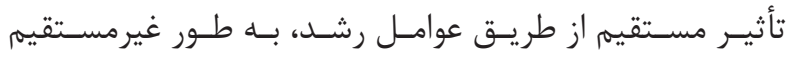

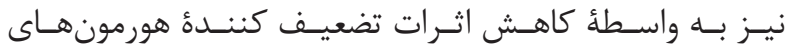

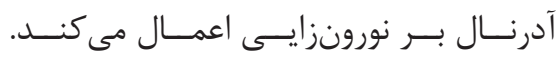

نورويتيتيدها

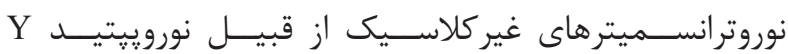

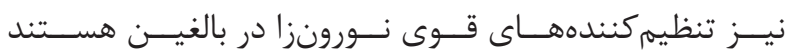

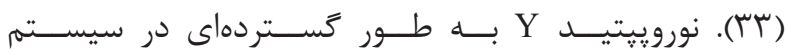

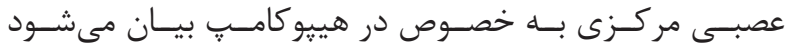

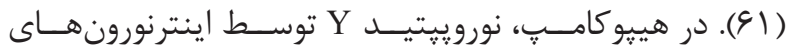

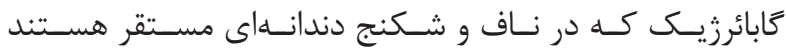

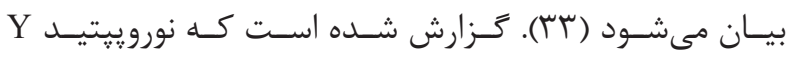

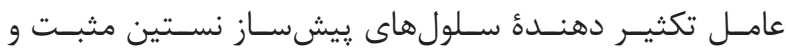

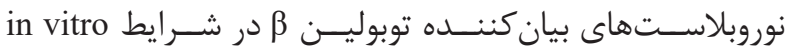

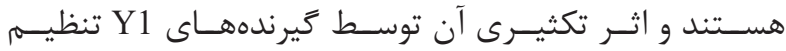

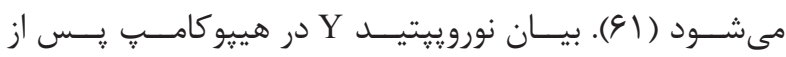

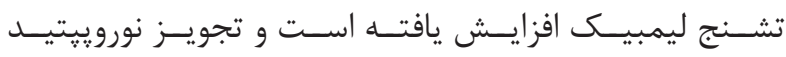

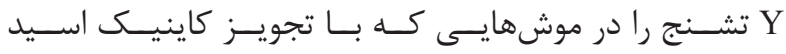

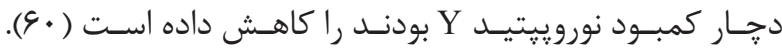

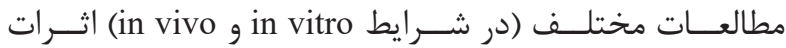

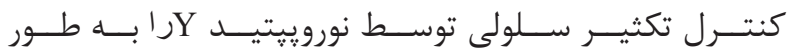

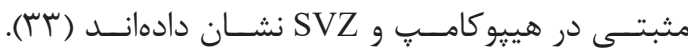

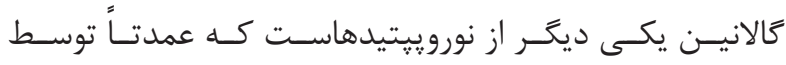

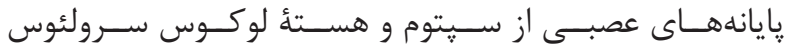

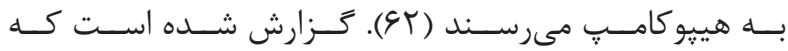

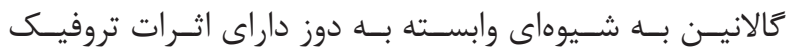

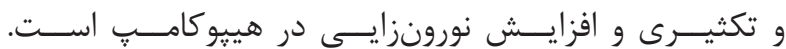

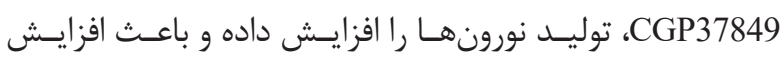

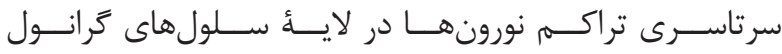

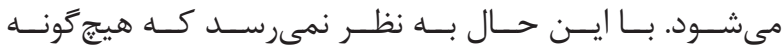

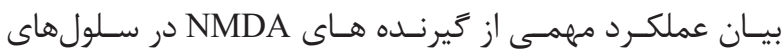

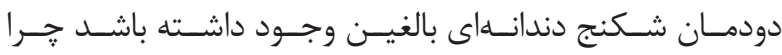

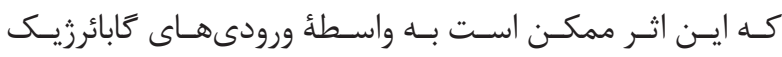

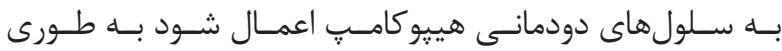

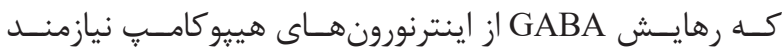

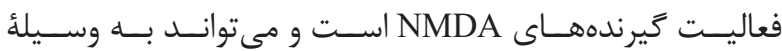

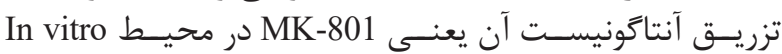

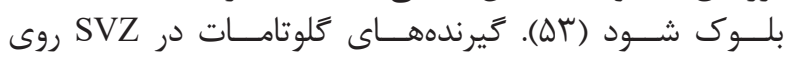

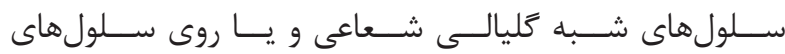

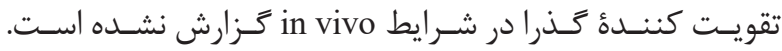

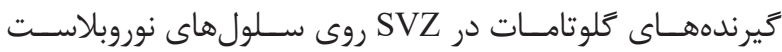

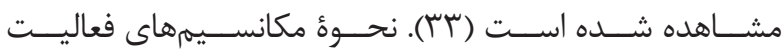

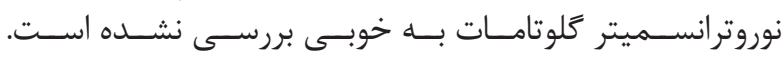

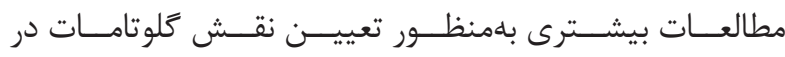

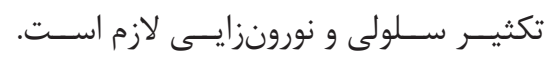

\section{كانابينوئيدها}

كانابينوئيدهـــا يــــ رده منحصــر بـــهـ فــرد از تركيبــات

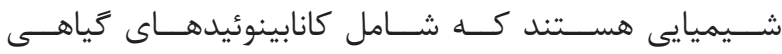

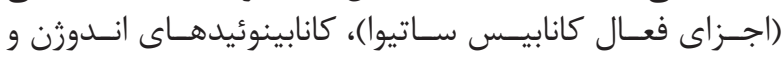

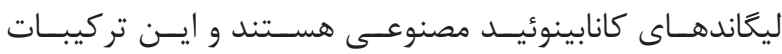

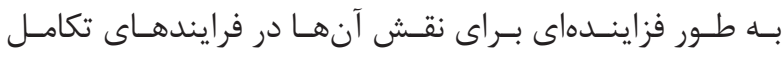

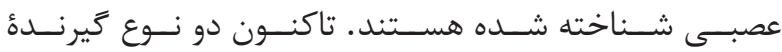

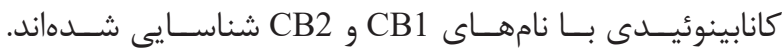

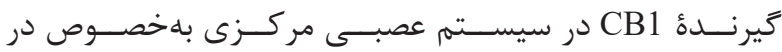

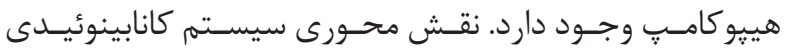

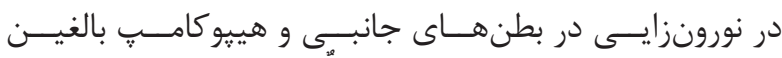

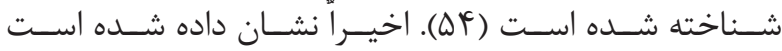

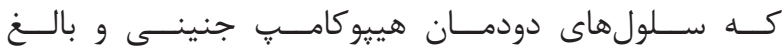

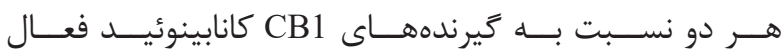

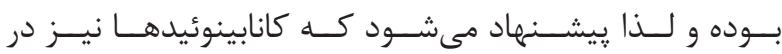

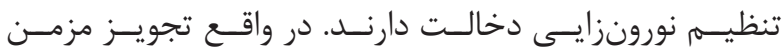

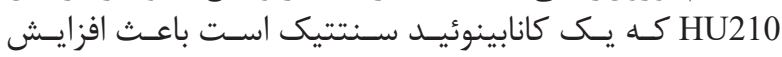

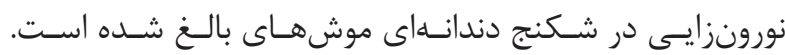

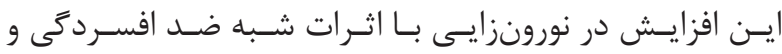

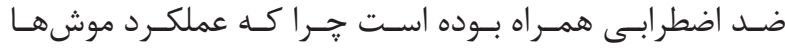

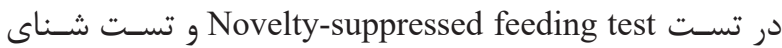

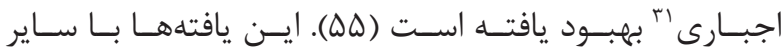

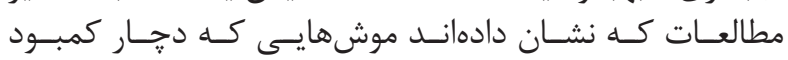

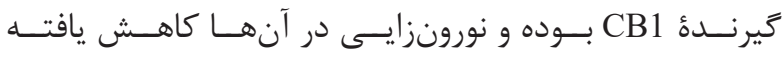

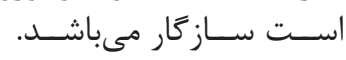

\section{كورتيكواستروئيدها}

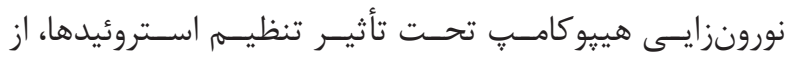

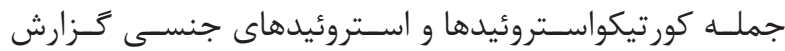

${ }^{31}$ Forced swim test 
بــه نورونزايسى در شــرايط پاتولوزيـــ مغــزى در مطالعـات

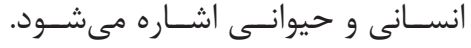

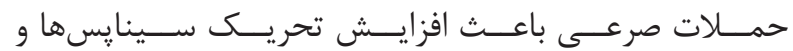

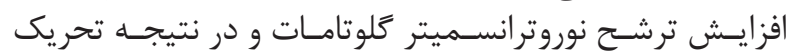

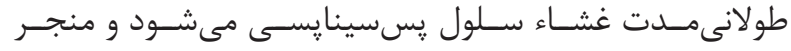

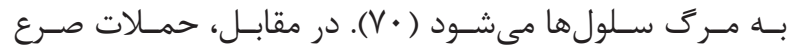

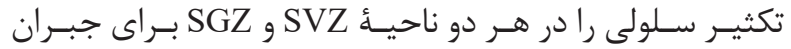

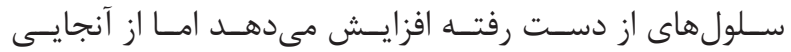

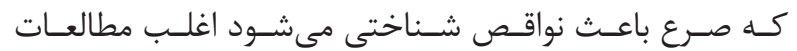

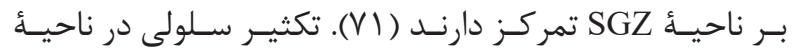

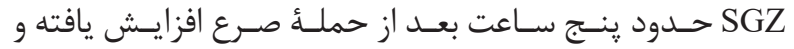

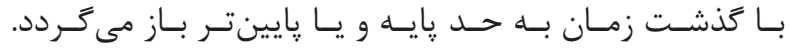

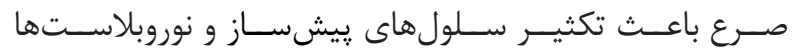

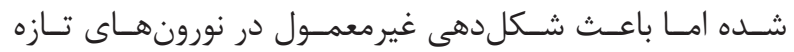

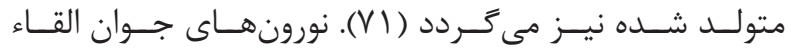

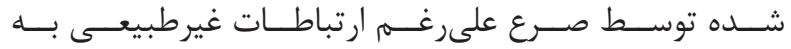

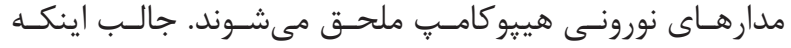

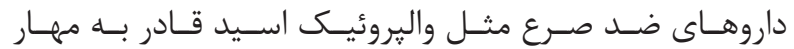

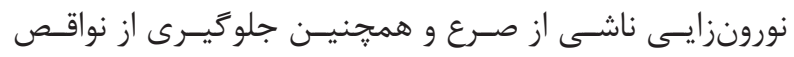

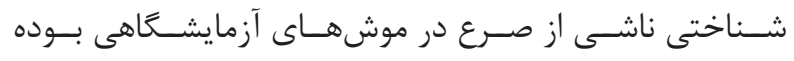

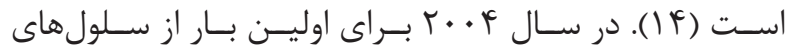

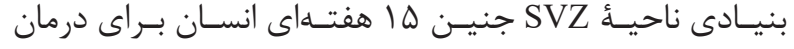

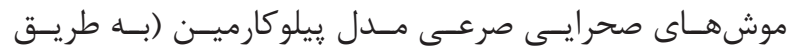

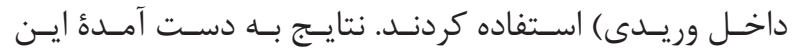

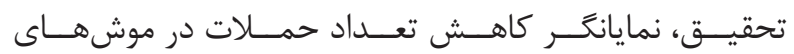

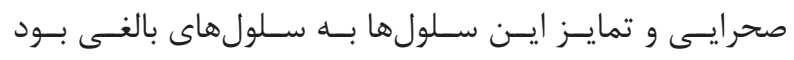

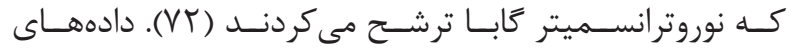

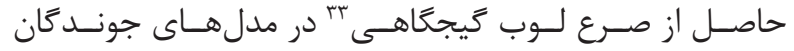

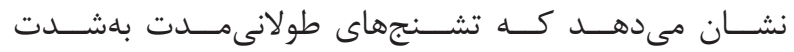

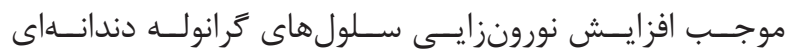

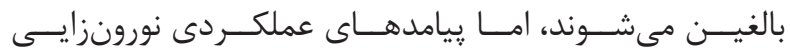

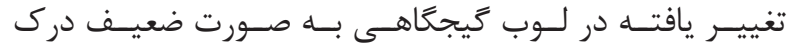

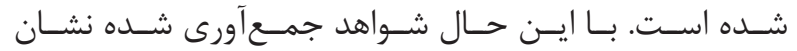

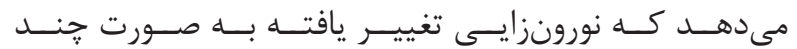

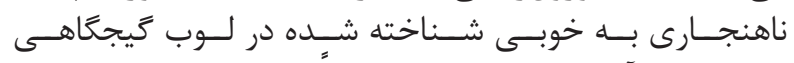

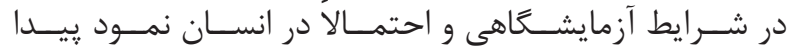

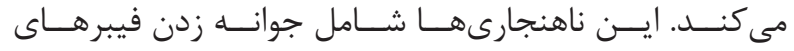

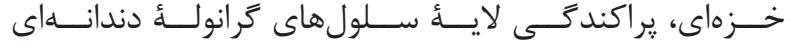

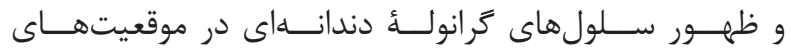

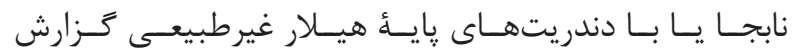

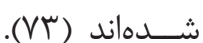

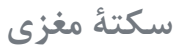

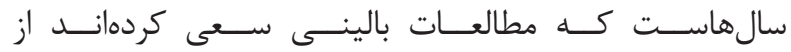

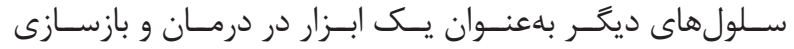

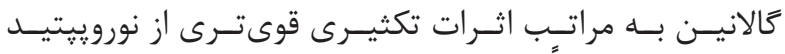

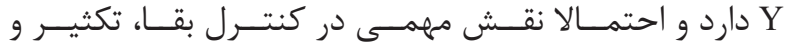

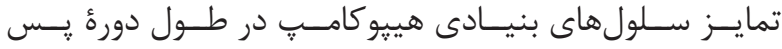

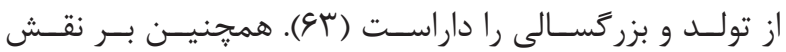

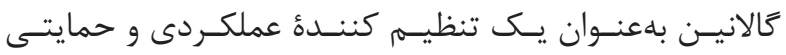

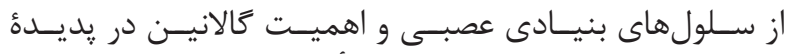

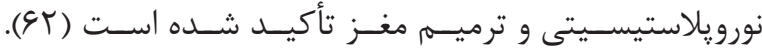

اهميت عملكردى نورونزايى

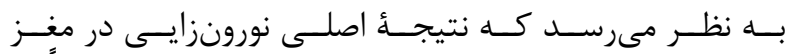

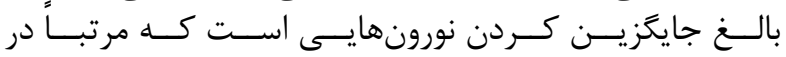

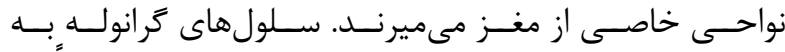

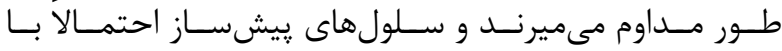

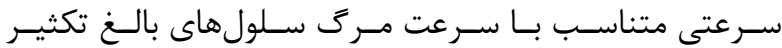

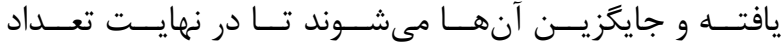

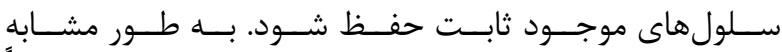

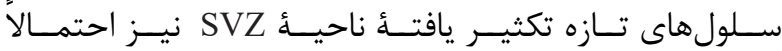

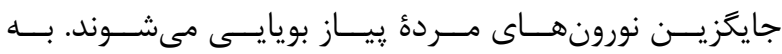

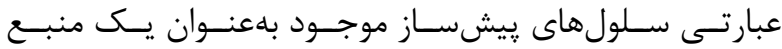

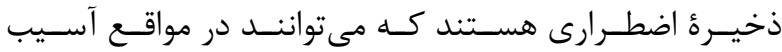

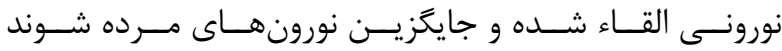

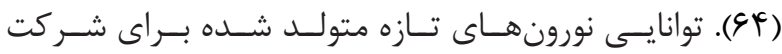

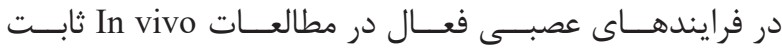

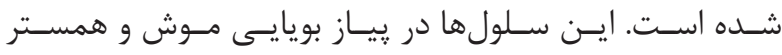

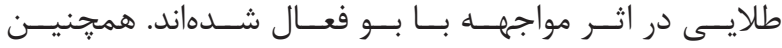

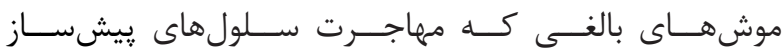

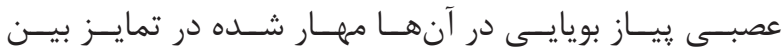

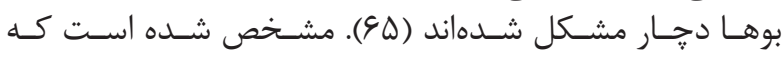

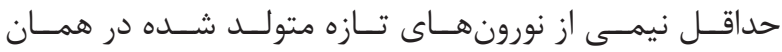

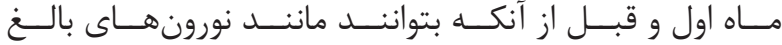

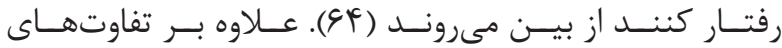

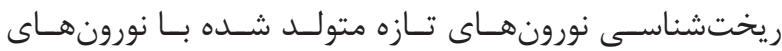

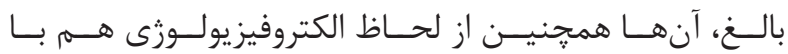

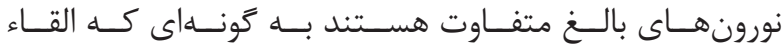

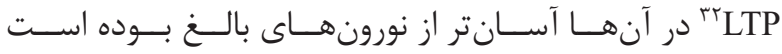

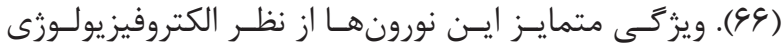

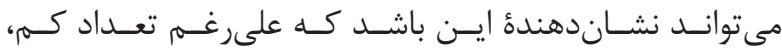

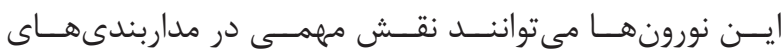

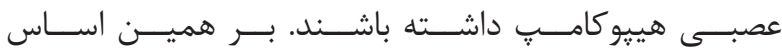

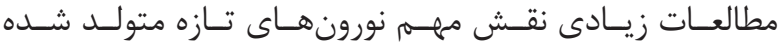

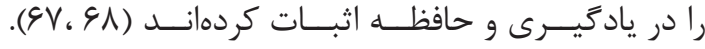

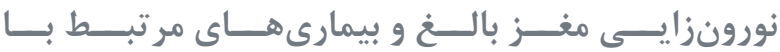

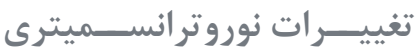

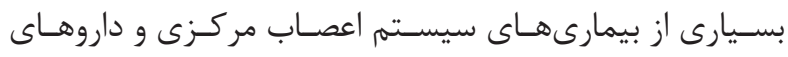

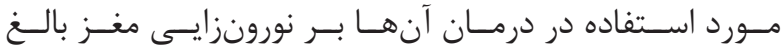

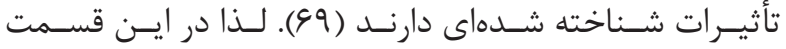

\footnotetext{
${ }^{32}$ Long-term potentiation

${ }^{33}$ Temporal lobe
} 


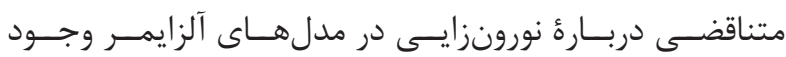

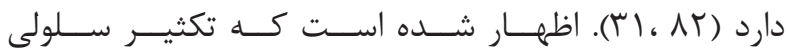

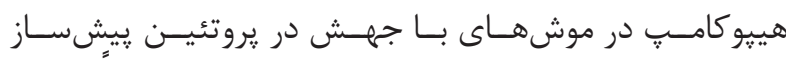

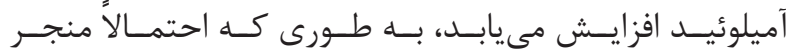

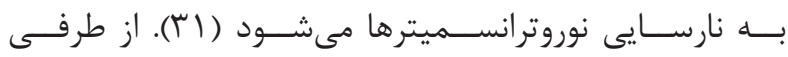

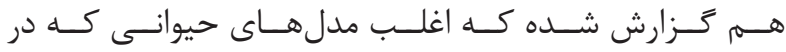

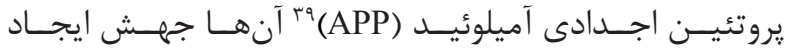

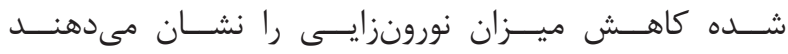

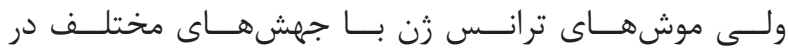

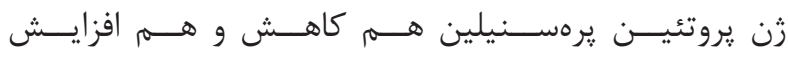

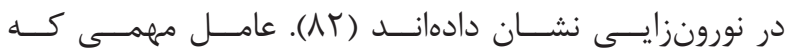

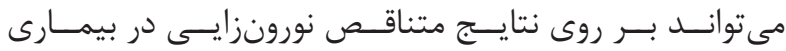

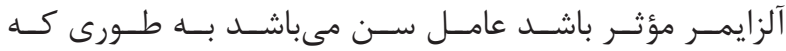

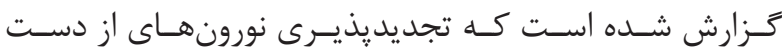

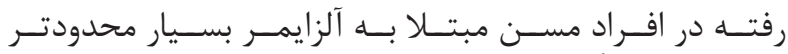

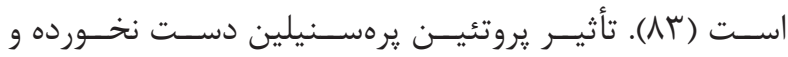

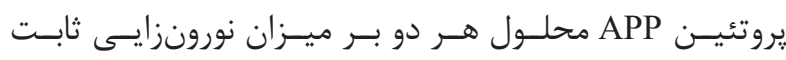

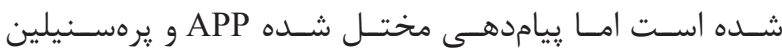

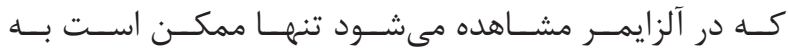

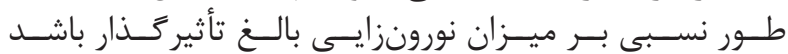

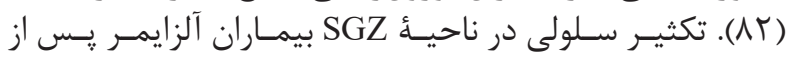

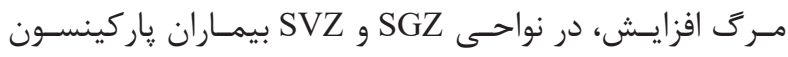

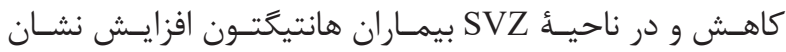

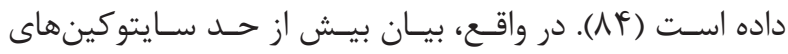

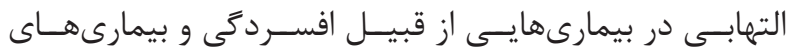

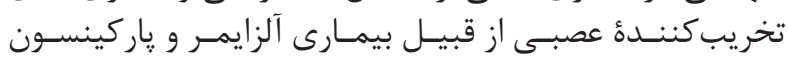

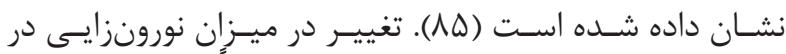

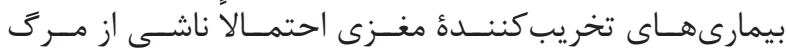

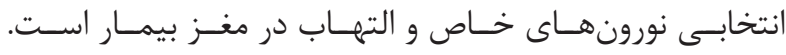

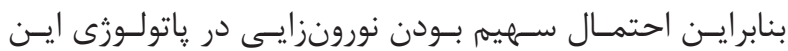

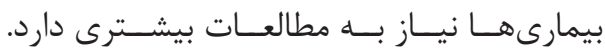

$$
\text { التهاب و ساير پاسخ هاى ايمنى بيماري }
$$

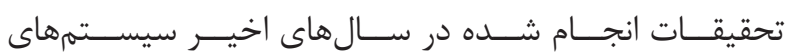

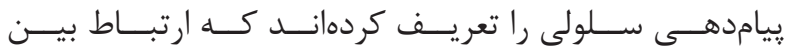

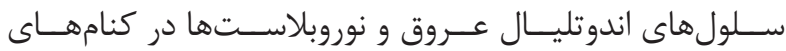

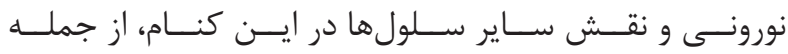

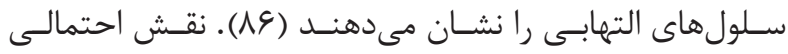

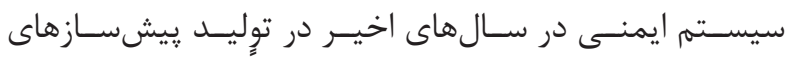

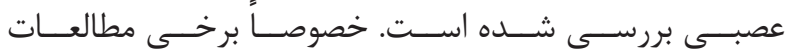

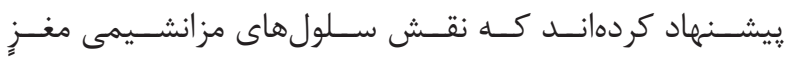

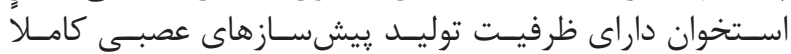

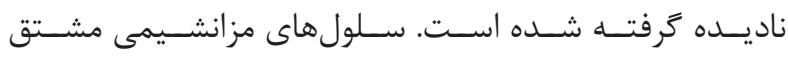

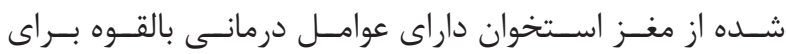

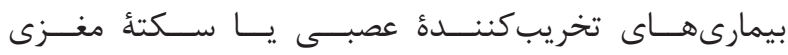

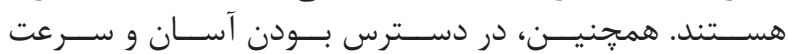

${ }^{34}$ Self-repair system

${ }^{35}$ Stroke

${ }^{36}$ Neurodegenerative diseases

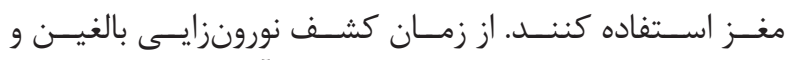

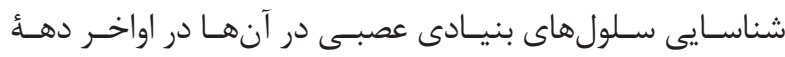

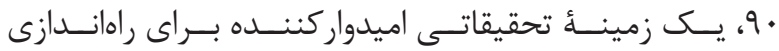

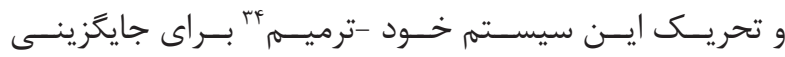

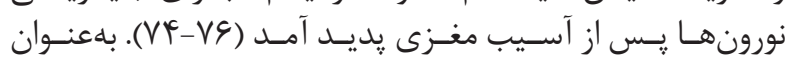

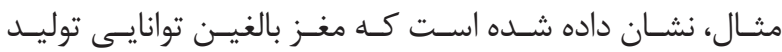

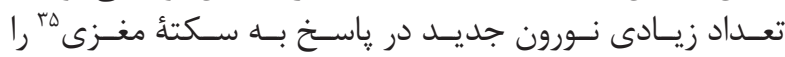

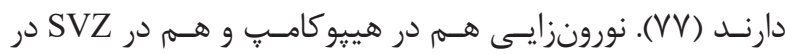

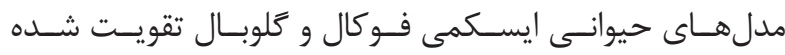

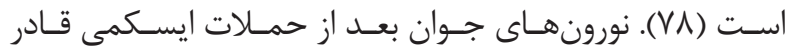

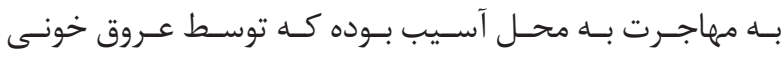

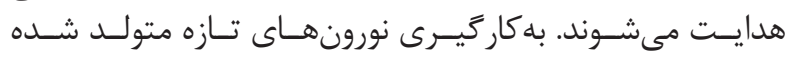

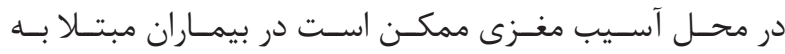

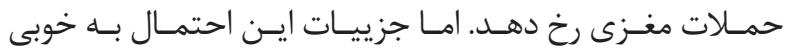

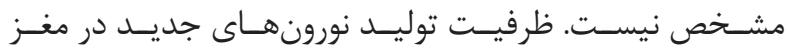

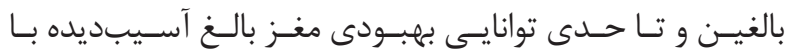

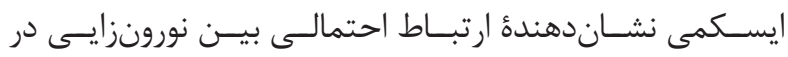

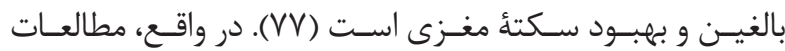

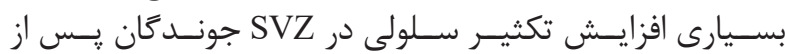

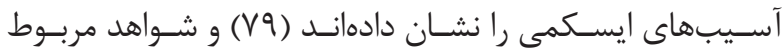

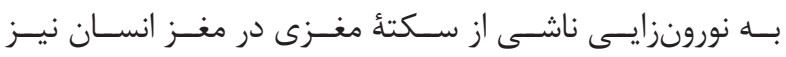

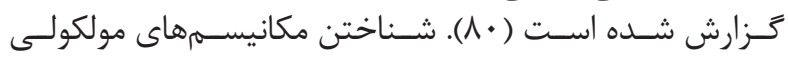

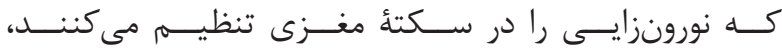

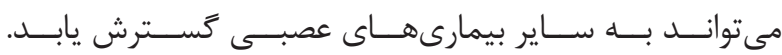

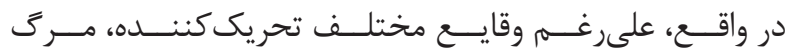

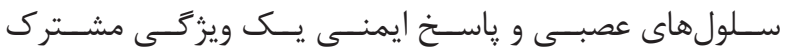

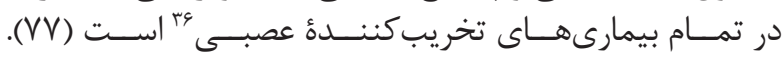

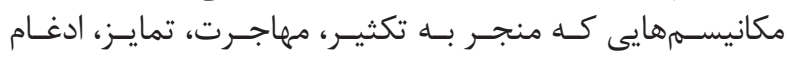

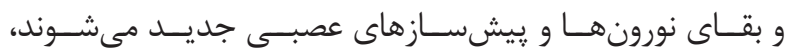

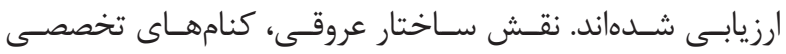

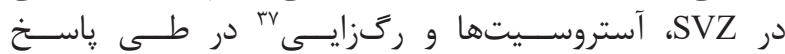

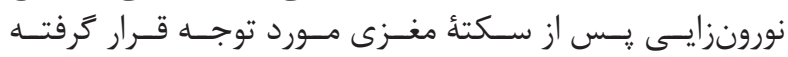

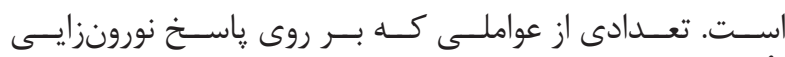

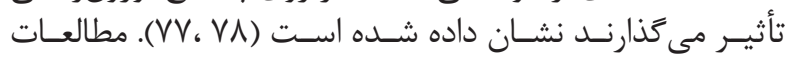

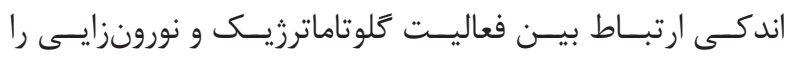

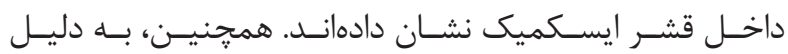

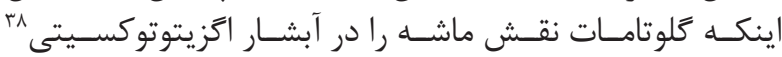

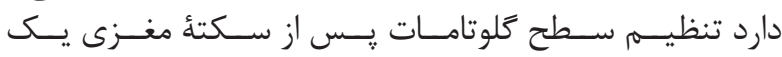

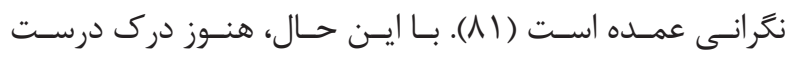

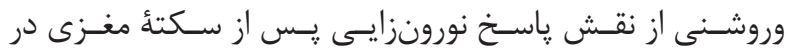

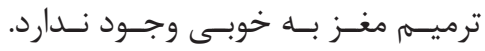

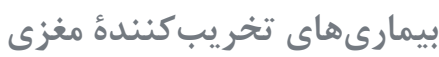

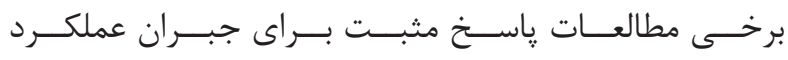

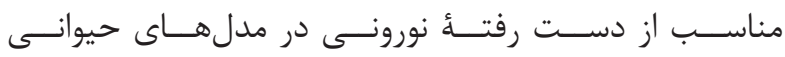

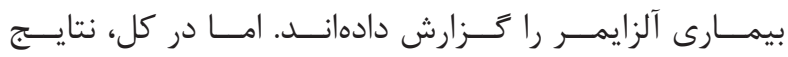

\footnotetext{
${ }^{37}$ Angiogenesis

${ }^{38}$ Excitotoxicity

${ }^{39}$ Amyloid precursor protein
} 


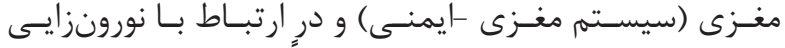

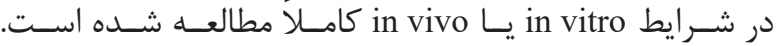

$$
\text { نتيجه }
$$

نورونزايسى در دو ناحيـه از مغـز يعنـى نواحسى SGZ و SVI

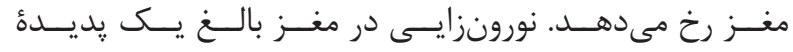

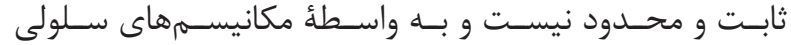

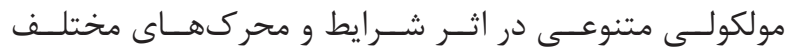

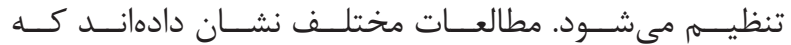

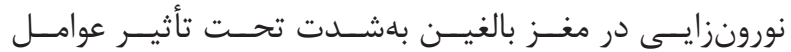

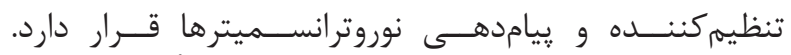

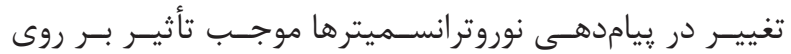

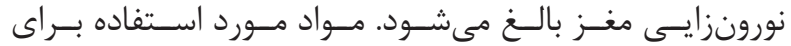

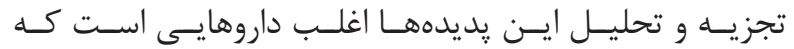

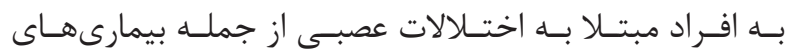

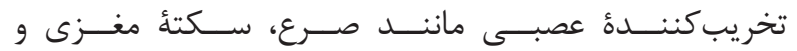

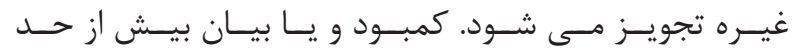

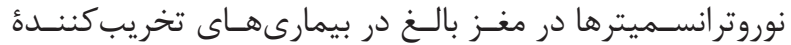

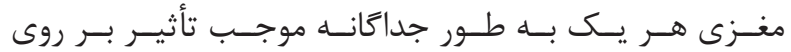

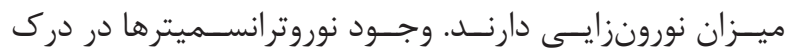

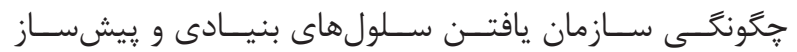

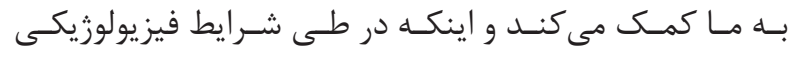

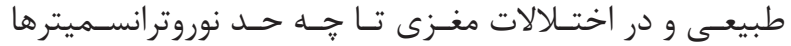

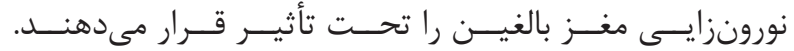

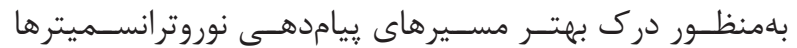

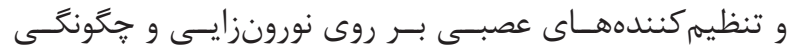

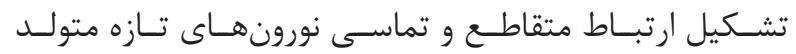

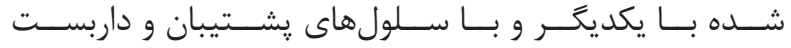

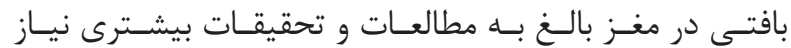

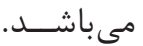

1. Cajal y, Santiago R. Cajal's degeneration and regeneration of the nervous system. New York: History of Neuroscience. 1991; p. 60-71.

2. Altman J. Are new neurons formed in the brains of adult mammals? Science. 1962; 135(3509): 1127-8.

3. Kaplan MS, Hinds JW. Neurogenesis in the adult rat: electron microscopic analysis of light radioautographs. Science. 1977; 197(4308): 1092-4.

4. Nottebohm F. A brain for all seasons: cyclical anatomical changes in song control nuclei of the canary brain. Science. 1981; 214(4527): 1368-70.

5. Marucci G. Commentary on human adult neurogenesis across the ages: an immunohistochemical study. Neuropathol Appl Neurobiol. 2017; 43(5): 450-1.

6. Sorrells SF, Paredes MF, Cebrian-Silla, Sandoval

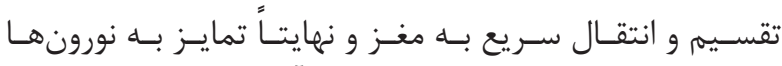

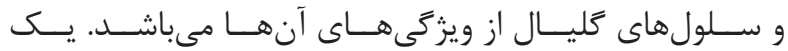

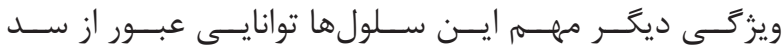

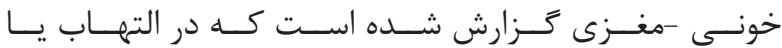

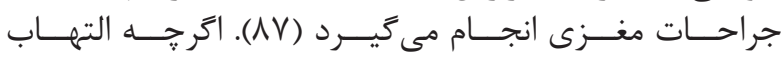

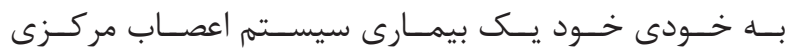

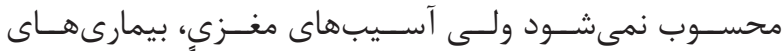

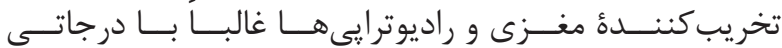

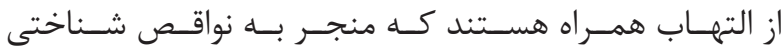

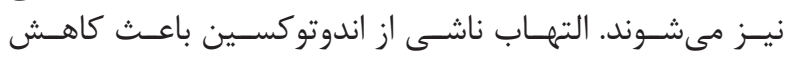

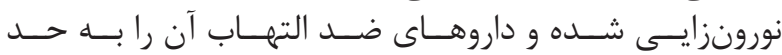

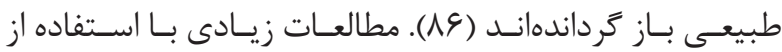

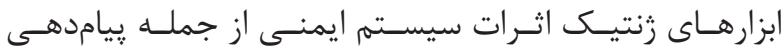

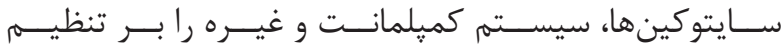

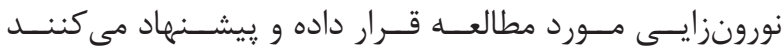

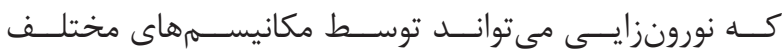

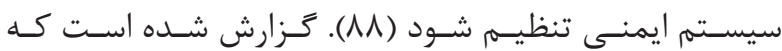

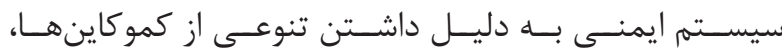

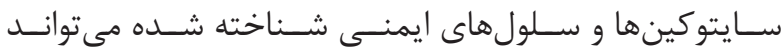

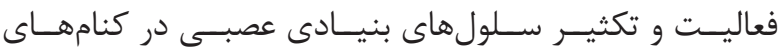

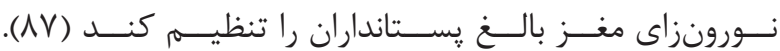

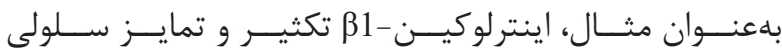

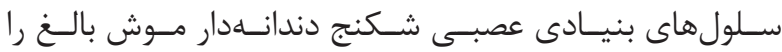

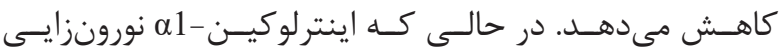

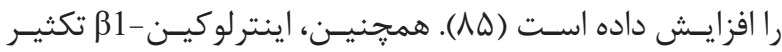

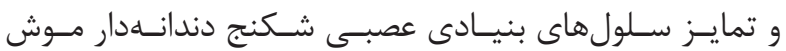

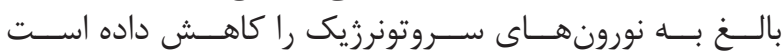

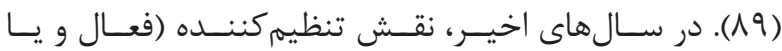

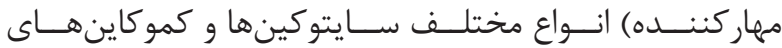

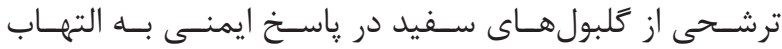

منابع

K, Qi D, Kelley KW, et al. Human hippocampal neurogenesis drops sharply in children to undetectable levels in adults. Nature. 2018; 555(7696): 377-81.

7. Ernst C, Christie BR. Temporally specific proliferation events are induced in the hippocampus following acute focal injury. J Neurosci Res. 2006; 83(3): 349-61.

8. Sahab Negah S, Mohammad Sadeghi S, Kazemi H, Modarres Mousavi M, Aligholi H. Effect of injured brain extract on proliferation of neural stem cells cultured in 3-dimensional environment. Shefaye Khatam. 2015; 3(1): 49-56.

9. Gustafsson E, Lindvall O, Kokaia Z. Intraventricular infusion of TrkB-Fc fusion protein promotes ischemiainduced neurogenesis in adult rat dentate gyrus. Stroke. 2003; 34(11): 2710-5.

10. Yoshimura S, Takagi Y, Harada J, Teramoto T, 
Thomas SS, Waeber C, et al. FGF-2 regulation of neurogenesis in adult hippocampus after brain injury. Proc Natl Acad Sci. 2001; 98(10): 5874-9.

11. Arvidsson A, Kokaia Z, Lindvall O. N-Methyl-Daspartate receptor-mediated increase of neurogenesis in adult rat dentate gyrus following stroke. Eur J Neurosci. 2001; 14(1): 10-8.

12. Clemenson GD, Deng W, Gage FH. Environmental enrichment and neurogenesis: from mice to humans. Curr Opin Behav Sci. 2015; 4: 56-62.

13. Kent BA, Mistlberger RE. Sleep and hippocampal neurogenesis: implications for Alzheimer's disease. Front Neuroendocrinol. 2017. 45:35-52.

14. Sahab Negah S, Khooei A, Samini F, Gorji A. Laminin-derived Ile-Lys-Val-Ala-Val: a promising bioactive peptide in neural tissue engineering in traumatic brain injury. Cell Tissue Res. 2018; 3371(2): 223-36.

15. Zhang Y, Kim MS, Jia B, Yan J, Zuniga-Hertz JP, Han C, et al. Hypothalamic stem cells control ageing speed partly through exosomal miRNAs. Nature. 2017; 548(7665): 52-7.

16. Zhao C, Deng W, Gage FH. Mechanisms and functional implications of adult neurogenesis. Cell. 2008; 132(4): 645-60.

17. Song H, Stevens CF, Gage FH. Astroglia induce neurogenesis from adult neural stem cells. Nature. 2002; 417(6884): 39-44.

18. Lim DA, Tramontin AD, Trevejo JM, Herrera DG, Garcia-Verdugo JM, Alvarez-Buylla A. Noggin antagonizes BMP signaling to create a niche for adult neurogenesis. Neuron. 2000; 28(3): 713-26.

19. Hoglinger GU, Rizk P, Muriel MP, Duyckaerts C, Oertel WH, Caille I, et al. Dopamine depletion impairs precursor cell proliferation in Parkinson disease. Nat Neurosci. 2004; 7(7): 726-35.

20. Sahab Negah S, Khaksar Z, Kazemi H, Aligholi H, Safahani M, Mostafa S, et al. The role of dopamine receptors during brain development. Shefaye Khatam. $2014 ; 2(3): 65-76$.

21. Alvarez-Buylla A, Lim DA. For the long run: maintaining germinal niches in the adult brain. Neuron. 2004; 41(5): 683-6.

22. Cao L, Jiao X, Zuzga DS, Liu Y, Fong DM, Young D, et al. VEGF links hippocampal activity with neurogenesis, learning and memory. Nat Genet. 2004; 36(8): 827-35

23. Khaksar Z, Sahab Negah S, Mohammad Sadeghi S. Effects of a self-assembling peptide nanofiber containing laminin motif on survival and proliferation of embryonic rat neural stem cells. Shefaye Khatam. 2016; 4(2): 55-64.

24. Suh H, Consiglio A, Ray J, Sawai T, D’Amour KA, Gage FH. In vivo fate analysis reveals the multipotent and self-renewal capacities of Sox2+ neural stem cells in the adult hippocampus. Cell Stem Cell. 2007; 1(5): 515-28.

25. Doetsch F, Caille I, Lim DA, Garcia-Verdugo JM, Alvarez-Buylla A. Subventricular zone astrocytes are neural stem cells in the adult mammalian brain. Cell. 1999; 97(6): 703-16.

26. Abrous DN, Koehl M, Le Moal M. Adult neurogenesis: from precursors to network and physiology. Physiol Rev. 2005; 85(2): 523-69.

27. Urban N, Guillemot F. Neurogenesis in the embryonic and adult brain: same regulators, different roles. Front Cell Neurosci. 2014; 8: 396. doi: 10.3389/ fncel.2014.00396.

28. Ihunwo AO, Tembo LH, Dzamalala C. The dynamics of adult neurogenesis in human hippocampus. Neural Regen Res. 2016; 11(12): 1869-83.

29. Yang T-T, Lo C-P, Tsai P-S, Wu S-Y, Wang T-F, Chen Y-W, et al. Aging and exercise affect hippocampal neurogenesis via different mechanisms. PloS One. 2015; 10(7): e0132152.

30. Azmitia EC, Gannon PJ, Kheck NM, WhitakerAzmitia PM. Cellular localization of the 5-HT1A receptor in primate brain neurons and glial cells. Neuropsychopharmacology. 1996; 14(1): 35-46.

31. Berg DA, Belnoue L, Song H, Simon A. Neurotransmitter-mediated control of neurogenesis in the adult vertebrate brain. Development. 2013; 140: 2548-61.

32. Alenina N, Klempin F. The role of serotonin in adult hippocampal neurogenesis. Behav Brain Res. 2015; 277: 49-57.

33. Kuhn HG. Control of cell survival in adult mammalian neurogenesis. Cold Spring Harb Perspect Biol. 2015; 7(12): 1-12.

34. Mohapel P, Leanza G, Kokaia M, Lindvall O. Forebrain acetylcholine regulates adult hippocampal 


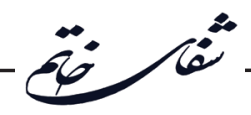

neurogenesis and learning. Neurobiol Aging. 2005; 26(6): 939-46.

35. Harrist A, Beech RD, King SL, Zanardi A, Cleary MA, Caldarone BJ, et al. Alteration of hippocampal cell proliferation in mice lacking the beta 2 subunit of the neuronal nicotinic acetylcholine receptor. Synapse. 2004; 54(4): 200-6.

36. Kulkarni VA, Jha S, Vaidya VA. Depletion of norepinephrine decreases the proliferation, but does not influence the survival and differentiation, of granule cell progenitors in the adult rat hippocampus. Eur J Neurosci. 2002; 16(10): 2008-12.

37. Pathania M, Yan LD, Bordey A. A symphony of signals conducts early and late stages of adult neurogenesis. Neuropharmacology. 2010; 58(6): 865-76.

38. Borta A, Hoglinger GU. Dopamine and adult neurogenesis. J Neurochem. 2007; 100(3): 587-95.

39. Pontes A, Zhang Y, Hu W. Novel functions of GABA signaling in adult neurogenesis. Front Biol. 2013; 8(5): 496-507.

40. Maekawa M, Namba T, Suzuki E, Yuasa S, Kohsaka $\mathrm{S}$, Uchino S. NMDA receptor antagonist memantine promotes cell proliferation and production of mature granule neurons in the adult hippocampus. Neurosci Res. 2009; 63(4): 259-66.

41. Brezun JM, Daszuta A. Serotonin may stimulate granule cell proliferation in the adult hippocampus, as observed in rats grafted with foetal raphe neurons. Eur J Neurosci. 2000; 12: 391-6.

42. Arnold SA, Hagg T. Serotonin 1A receptor agonist increases species- and region-selective adult CNS proliferation, but not through CNTF. Neuropharmacology. 2012; 63: 1238-47.

43. Mohapel P, Leanza G, Kokaia M, Lindvall O. Forebrain acetylcholine regulates adult hippocampal neurogenesis and learning. Neurobiol Aging. 2005; 26: 939-46.

44. Van Kampen JM, Eckman CB. Agonist-induced restoration of hippocampal neurogenesis and cognitive improvement in a model of cholinergic denervation. Neuropharmacology. 2010; 58(6): 921-9.

45. Kulkarni VA, Jha S, Vaidya VA. Depletion of norepinephrine decreases the proliferation, but does not influence the survival and differentiation, of granule cell progenitors in the adult rat hippocampus. Eur $\mathrm{J}$ Neurosci. 2002; 16(10): 2008-12.
46. Jhaveri DJ, Mackay EW, Hamlin AS, Marathe SV, Nandam LS, Vaidya VA, et al. Norepinephrine directly activates adult hippocampal precursors via beta3adrenergic receptors. J Neurosci. 2010; 30: 2795-806.

47. Parish CL, Beljajeva A, Arenas E, Simon A. Midbrain dopaminergic neurogenesis and behavioral recovery in a salamander lesion induced regeneration model. Development. 2007; 134: 2881-7.

48. Neve KA, Seamans JK, Trantham-Davidson H. Dopamine receptor signaling. J Recept Signal Transduct Res. 2004; 24(3): 165-205.

49. Halim ND, Weickert CS, McClintock BW, Weinberger DR, Lipska, BK. Effects of chronic haloperidol and clozapine treatment on neurogenesis in the adult rat hippocampus. Neuropsychopharmacology. 2004; 29(6): 1063-9.

50. Dawirs RR, Hildebrandt K, Teuchert-Noodt G. Adult treatment with haloperidol increases dentate granule cell proliferation in the gerbil hippocampus. J Neural Transm. 1998; 105(2-3): 317-27.

51. Song J, Zhong C, Bonaguidi MA, Sun GJ, Hsu D, $\mathrm{Gu} \mathrm{Y}$, et al. Neuronal circuitry mechanism regulating adult quiescent neural stem-cell fate ecision. Nature. 2012; 489(7414): 150-4.

52. Howell OW, Silva S, Scharfman HE, Sosunov AA, Zaben M, Shtaya A, et al. Neuropeptide Y is important for basal and seizure-induced precursor cell proliferation in the hippocampus. Neurobiol Dis. 2007; 26(1): 174-88.

53. Grote HE, Hannan AJ. Regulators of adult neurogenesis in the healthy and diseased brain. Clin Exp pharmacol Physiol. 2007; 34(5-6): 533-45.

54. Prenderville JA, Kelly AM, Downer EJ. The role of cannabinoids in adult neurogenesis. Br J Pharmacol. 2015; 172(16): 3950-63.

55. Jiang W, Zhang Y, Xiao L, Van Cleemput J, Ji S-P, Bai $\mathrm{G}$, et al. Cannabinoids promote embryonic and adult hippocampus neurogenesis and produce anxiolytic-and antidepressant-like effects. J Clin Invest. 2005; 115(11): 3104-16.

56. Lieberwirth C, Pan Y, Liu Y, Zhang Z, Wang Z. Hippocampal adult neurogenesis: Its regulation and potential role in spatial learning and memory. Brain Res. 2016; 1644: 127-40.

57. Kim JB, Ju JY, Kim JH, Kim TY, Yang BH, Lee YS, et al. Dexamethasone inhibits proliferation of adult hippocampal neurogenesis in vivo and in vitro. Brain 
Res. 2004; 1027 (1-2): 1-10.

58. Spanswick S, Epp J, Sutherland R. Time-course of hippocampal granule cell degeneration and changes in adult neurogenesis after adrenalectomy in rats. Neuroscience. 2011; 190: 166-76.

59. Cameron HA, Tanapat P, Gould E. Adrenal steroids and N-Methyl-D-aspartate receptor activation regulate neurogenesis in the dentate gyrus of adult rats through a common pathway. Neuroscience. 1998; 82(2): 349-54.

60. Fairhurst GD, Frey MD, Reichert JF, Szelest I, Kelly DM, Bortolotti GR. Does environmental enrichment reduce stress? An integrated measure of corticosterone from feathers provides a novel perspective. PloS One. 2011; 6(3): e17663.

61. Howell OW, Scharfman HE, Herzog H, Sundstrom LE, Beck-Sickinger A, Gray WP. Neuropeptide Y is neuroproliferative for post-natal hippocampal precursor cells. J Neurochem. 2003; 86: 646-59.

62. Cordero-Llana O, Rinaldi F, Brennan PA, Wynick D, Caldwell MA. Galanin promotes neuronal differentiation from neural progenitor cells in vitro and contributes to the generation of new olfactory neurons in the adult mouse brain. Exp Neurol. 2014; 256: 93-104.

63. Zaben MJ, Gray WP. Neuropeptides and hippocampal neurogenesis. Neuropeptides. 2013;47(6):431-8.

64. Braun SM, Jessberger S. Adult neurogenesis: mechanisms and functional significance. Development. 2014; 141(10): 1983-6.

65. Whitman MC, Greer CA. Adult neurogenesis and the olfactory system. Prog Neurobiol. 2009; 89(2): 162-75.

66. Kempermann G, Wiskott L, Gage FH. Functional significance of adult neurogenesis. Curr Opin Neurobiol. 2004; 14(2): 186-91.

67. Anacker C, Hen R. Adult hippocampal neurogenesis and cognitive flexibility - linking memory and mood. Nat Rev Neurosci. 2017; 18(6): 335-46.

68. Baghishani F, Sahab Negah S. The role of neurogenesis in anxiety disorders. Shefaye Khatam. 2017; 5(2): 98-109.

69. Pasand Mozhdeh H, Zeynali B, Aligholi H, Kashani Radgerdi I, Sahab Negah S, Hassanzadeh G. The effect of intracerebroventricular administration of streptozocin on cell proliferation in subventricular zone stem cells in a rat model of alzheimer's disease. Shefaye Khatam. 2015; 3(4): 80-6.
70. Rigoulot MA, Koning E, Ferrandon A, Nehlig A. Neuroprotective properties of topiramate in the lithiumpilocarpine model of epilepsy. J Pharmacol Exp Ther. 2004; 308(2): 787-95.

71. Jessberger S, Parent JM. Epilepsy and adult neurogenesis. Cold Spring Harb Perspect Biol. 2015; 7(12): a020677.

72. Chu K, Kim M, Jung KH, Jeon D, Lee ST, Kim J, et al. Human neural stem cell transplantation reduces spontaneous recurrent seizures following pilocarpineinduced status epilepticus in adult rats. Brain Res. 2004; 1023(2): 213-21.

73. Parent JM, Kron MM. Neurogenesis and Epilepsy. Noebels JL, Avoli M, Rogawski MA. Jasper's Basic Mechanisms of the Epilepsies. 4nd ed. US: Bethesda (MD): National Center for Biotechnology Information. 2012; p. 56-61.

74. Sahab Negah S, Aligholi H, Khaksar Z, Kazemi H, Mousavi SM, Safahani M, et al. Survival, proliferation, and migration of human meningioma stem-like cells in a nanopeptide scaffold. Iran J Basic Med Sci. 2016; 19(12): 1271-8.

75. Jahanbazi Jahan-Abad A, Morteza-Zadeh P, Sahab Negah S, Gorji A. Curcumin attenuates harmful effects of arsenic on neural stem/progenitor cells. Avicenna J Phytomed. 2017; 7(4): 376-88.

76. Sahab Negah S, Khaksar Z, Aligholi H, Mohammad Sadeghi S, Modarres Mousavi SM, Kazemi H, et al. Enhancement of neural stem cell survival, proliferation, migration, and differentiation in a novel self-assembly peptide nanofibber scaffold. Mol Neurobiol. 2017. 54(10): 8050-62.

77. Marlier Q, Verteneuil S, Vandenbosch R, Malgrange, B. Mechanisms and functional significance of strokeinduced neurogenesis. Front Neurosci. 2015; 9: 458-74.

78. Lindvall O, Kokaia Z. Neurogenesis following stroke affecting the adult brain. Cold Spring Harb Perspect Biol. 2015; 7(11): a019034.

79. Thored P, Arvidsson A, Cacci E, Ahlenius H, Persistent production of neurons from adult brain stem cells during recovery after stroke. Stem Cells. 2006; 24(3): 739-47.

80. Jin K, Wang X, Xie L, Mao XO, Zhu W. Evidence for stroke-induced neurogenesis in the human brain. Proc Natl Acad Sci. 2006; 103(35): 13198-202.

81. Sanchez-Mendoza E, Bellver-Landete V, Merino JJ, Gonzalez MP, Martínez-Murillo R, Oset-Gasque MJ. 
Could neurotransmitters influence neurogenesis and neurorepair after stroke? Neuropathol Appl Neurobiol. 2013; 39(7): 722-35.

82. Mu Y, Gage FH. Adult hippocampal neurogenesis and its role in Alzheimer's disease. Mol Neurodegener. 2011; 6(85). doi: 10.1186/1750-1326-6-85.

83. Jin K, Peel AL, Mao XO, Xie L, Cottrell BA, Henshall DC, et al. Increased hippocampal neurogenesis in Alzheimer's disease. Proc Natl Acad Sci. 2004; 101(1): 343-7.

84. Winner B, Winkler J. Adult neurogenesis in neurodegenerative diseases. Cold Spring Harb Perspect Biol. 2015; 7(4): a021287.

85. Borsini A, Zunszain PA, Thuret S, Pariante CM. The role of inflammatory cytokines as key modulators of neurogenesis. Trends Neurosci. 2015; 38(3): 145-57.

86. Ekdahl CT, Claasen J-H, Bonde S, Kokaia Z, Lindvall O. Inflammation is detrimental for neurogenesis in adult brain. Proc Natl Acad Sci. 2003; 100(23): 13632-7.

87. Steffen H, Oliver von BH. A possible role for the immune system in adult neurogenesis: new insights from an invertebrate model. Zoology. 2016; 119: 153-7.

88. De Miranda AS, Zhang C-J, Katsumoto A, Teixeira AL. Hippocampal adult neurogenesis: does the immune system matter? J Neurol Sci. 2017; 372: 482-95.

89. Zhang K, Xu H, Cao L, Li K, Huang Q. Interleukin$1 \mathrm{~b}$ inhibits the differentiation of hippocampal neural precursor cells into serotonergic neurons. Brain Res. 2013; 1490: 193-201. 